\title{
Pollution load assessment and potential environmental risks of composite industrial effluents discharged from SIIDCUL Integrated Industrial Estate, Haridwar (Uttarakhand), India
}

Authors Info

T. Arora ${ }^{1 *}$, A. Mishra', G. Matta', A. K. Chopra', A. Kumar', D.R. Khanna' and V. Kumar

'Department of Zoology and Environmental Science, Gurukul Kangri University, Haridwar-249 404, India

${ }^{2}$ Department of Mathematics and Statistics, Gurukul Kangri University, Haridwar-249 404, India

${ }^{*}$ Corresponding Author Email : tushararora09@rediffmail.com

Key words

Groundwater Haridwar,

Heavy metals, Industrial effluent,

Physico-chemical parameters

Publication Info

Paper received : 11.07 .2015

Revised received: 08.01 .2016

Re-revised received : 14.02 .2016

Accepted: 19.03 .2016
Abstract

Aim: Water pollution in unarguably one of the most fundamental environmental issue at global and local level. The industrial wastewaters are emerging as challenging threat to ecological balance. The aim of the present study was to investigate the pollution load of composite industrial effluents discharged from the State Infrastructure and Industrial Development Corporation of Uttarakhand Limited (SIIDCUL) Integrated Industrial Estate (IE) at Haridwar (Uttarakhand), India with reference to ecological risks.

Methodology: Due to large outstretch of industrial area, the industrial complex was divided into four clusters (Cluster A to Cluster D). The effluent samples were collected from the drainage channels marked from the sampling sites at industrial area. Standard methods of APHA (2012) were followed for the analysis of effluents samples.

Results: The results revealed that there was a remarkably high pollution load at industrial estate. The effluents discharged from the industrial units at Cluster $\mathrm{C}$ were slightly acidic ( $\mathrm{pH} 5.33$ ) and the parameters like TDS (2107.67 mg l-1), BOD (453.33 $\left.\mathrm{mg} \mathrm{l}^{-1}\right), \mathrm{COD}\left(1003.3 \mathrm{mgl}^{-1}\right)$ and some heavy metals like Fe (37.38 $\left.\mathrm{mg} \mathrm{l}{ }^{-1}\right), \mathrm{Cr}\left(11.254 \mathrm{mg} \mathrm{l}^{-1}\right), \mathrm{Cu}\left(4.9 \mathrm{mg} \mathrm{l}^{-1}\right)$ and $\mathrm{Pb}$ (2.11 $\mathrm{mg} \mathrm{l}^{-1}$ ) exceeded the permissible limits as prescribed by Bureau of Indian Standards (BIS, 2012), thereby indicating a considerably higher load of pollutants. The load of metals concentration in the effluents of different clusters was found in the following order: $\mathrm{Fe}>\mathrm{Cd}>\mathrm{Zn}>\mathrm{Ni}>\mathrm{Cr}>\mathrm{Cu}>\mathrm{Pb}>\mathrm{Mn}$. The higher content of metals in industrial wastewater may be due to the discharge of untreated effluents from metal processing industries.

Interpretation: Disposal of effluents can be a source of severe ecological risks in the form of contamination of groundwater, soil, vegetation. Consumption of crops and vegetables irrigated with these effluents may have serious threats to human health, thereby causing a risk of various ailments like encephalopathy, cardiovascular and kidney diseases; muscular pain and intestinal hemorrhage. It is recommended that pollution control and regulatory authorities should periodically monitor the quality of effluents so as to ensure the compliance of prescribed standards.

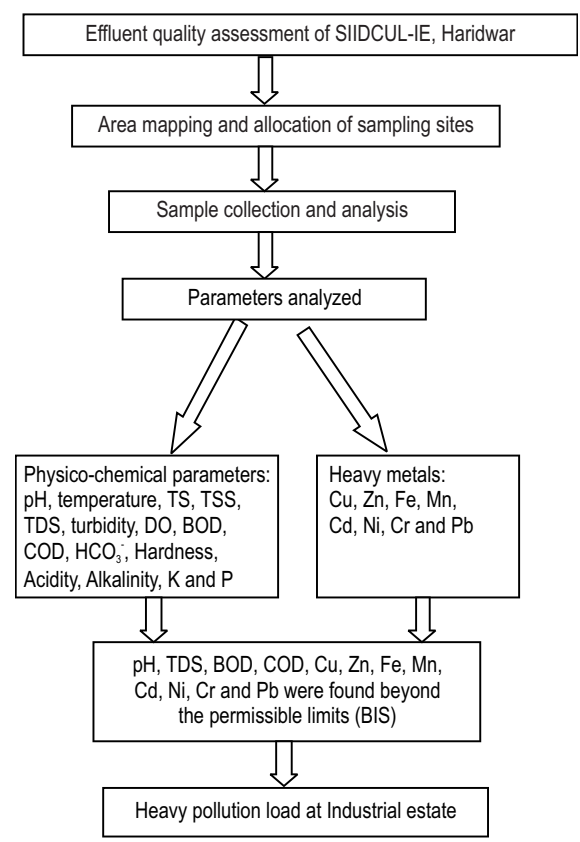




\section{Introduction}

Expanding population, rapid industrialization and pacing urbanization are responsible factors for environmental contamination. Environmental decontamination seems an enigma. In developing economies like India, environmental degradation is now being looked-up as a facet of economic upgradation. India is said to be in the first phase where it is experiencing the structural shift from agriculture to industrial growth (Krishna, 2007). As a consequence of this transition, large volumes of untreated industrial effluent are released in to open streams that deteriorate the surface water quality. When these toxic discharges are disposed off on land surface, they infiltrate into aquifers resulting into contaminated groundwater reserves (Jadhav and Nandan, 2016). Globally, it is estimated that industries dump 300-400 million tons of toxic metals, solvents, hazardous sludges, and other wastes into waters every year (United Nations Environment Program, 2010).

Contamination of groundwater resources by industrial wastewater has appeared as a new challenge that is being faced due to the emerging entrepreneurs. This feature is deteriorating the drinking water quality and posing major impact on soil system and crop productivity (Sahu et al., 2007; Arora et al., 20014; Mahmoud and Ghoneim, 2016). Morais et al. (2012) stated that industrial wastewater and solid wastes are regularly being discharged into the environment without any proper treatment. The toxic pollutants enter subsurface aquifers as a result of runoff and leaching process, which may ultimately pose health hazards among the local human and livestock population (Singh et al., 2007 and Raikwar et al., 2008).

High intensity of health disorders due to water borne diseases is well known in India. In many parts of the country, drinking water supplied from groundwater resources is plagued with quality crisis (CGWB, 2002). Till date, safe drinking water remains an imperative requisite, as $30 \%$ of urban and $90 \%$ of rural population still depend completely on untreated surface or groundwater (Kumar et al., 2005; Dutta and Ghosh, 2013; Islam et al., 2014 and Katiyar and Singh, 2014). According to Nickson et al. (2007) near by 66 million people in 22 Indian states are at risk due to elevated level of fluoride and around 10 million people of 6 states are under threat due to high concentration of arsenic in groundwater.

Apart from disposal of industrial wastewater on land and/or aquatic bodies, untreated effluents are also directly being injected into subsurface zones through ditches and injection wells, in order to avoid treatment and pollution abatement expenses (Peterson and Oberdorfer, 1985). Heavy metals are capable of immobilization in soil depending on soil pH. As a result, a small proportion of the overall concentration may leach to vadose-zone and groundwater resources of adjoining areas may become unsuitable for agriculture and/or drinking purposes
(Santona et al., 2006). Many researchers have investigated diffusion and dispersion process of heavy metals within the soil profile and their trend of migration in sub-surface layers along with interference of organic pollutants (Cuevas et al., 2011; Okereke, 2016).

In biotic perspective, heavy metals enter into living organisms through food, water or frequent contact to emission sources. Though few of them serve as vital micro nutrients for living beings but at higher concentration, they can lead to severe poisoning (Lenntech, 2004). Several toxic elements like lead, mercury and zinc are also well known neurological and endocrine disruptors. Toxicity of these elements chiefly involves brain and kidney ailments, but other symptoms may also appear (Neustadt and Pieczenik, 2007).

In the recent past, various studies regarding characterization of effluents of various industrial areas have also been made viz. at Kot Lakhpat industrial area, Lahore, Pakistan by Azeem (2009); Lokhande et al. (2011) at Taloja industrial area, Mumbai, India Nigeria; Joshi and Santani (2012) at Vapi India; Bharti et al. (2013) at Panipat, India; Idris et al. (2013) at Niger State, Nigeria; James et al. (2013) at Ogun State, Nigeria; Ramola and Singh (2013) at Dehradun, India; Singare and Dhabarde (2014) at Mumbai, India and Chuma et al. (2015) at Arusha City, Tanzania. Industrial estates normally have heterogeneous factories with varying pollution load and concentration.

The SIIDCUL Industrial Estate (SIIDCUL-IE) was established in 2006 in Haridwar district of Uttarakhand province in India. Within the last decade, a large number of industries have been established in this region including cosmetics, plastic, apparel, agro food, pharma-products, electric and electronic products, packaging, synthetic fabrics, electroplating, commercial automotives etc., but these industries lack individual wastewater treatment systems.

In context to newly installed industrial complex at Haridwar, no significant study concerning the assessment of pollution load due to discharged industrial effluents has been carried out till date. Keeping the above in view, present investigation was conducted to assess the pollution load and potential environmental risks of industrial effluents discharged from SIIDCUL Industrial Estate, Haridwar (Uttarakhand), India. The present study would be helpful to design appropriate treatment technologies and formulate the policies to control unscientific disposal of industrial effluents.

\section{Materials and Methods}

Study area: The industrial complex of SIIDCUL-IE, Haridwar (Uttarakhand), India (Latitude 29 ${ }^{\circ} 4^{\prime}$ ' north and Longitude $78^{\circ} 16^{\prime}$ ) is stretched in an area of about 2034 acres having nearly 700 




$\square=$ Effluent sampling site $\longrightarrow=$ Direction of flow of effluents
$=\mathrm{CA} \square=\mathrm{CB} \square=\mathrm{CD}$

Fig. 1 : Sampling sites at SIIDCUL-IE area at Haridwar, India

independent industrial units in operation. Major industries established at SIIDCUL industrial complex are shown in Fig. 1. In view of a large expanse of industrial complex, the SIIDCUL-IE was divided into four Clusters named as Cluster A(CA), Cluster B (CB), Cluster C (CC) and Cluster D (CD). At each sampling station, three sampling sites were marked for sampling of industrial effluents, thus having a total number of twelve sampling sites at industrial complex (Fig. 1). This differentiation was done on the basis of density of established industries in each cluster.

Sampling and characterization of effluents : Composite effluent samples were taken from the marked sampling sites (open as well as from covered drains of the effluent drainage system) delineated outside the premises of different industries. Monthly sampling was carried out during February 2013 to January 2014. The samples were collected in pre-washed polythene bottles of 3.0 liter capacity; between 9.00 a.m. to 12.00 p.m. The precautions were taken as per the standard guidelines to avoid any possible contamination. Estimation of different parameters was done by calibrated instruments viz., pH and total dissolved solids (TDS) by Microprocessor based digital water and soil analysis kit (ESICO, Model-1160), turbidity (NTU) by Turbidity meter (ESICO, Model-335), potassium by Microprocessor based flame photometer (ESICO, Model-1382) and phosphorus by UVVis Spectrophotometer (Agilent, Model- Cary 60). Other parameters like, dissolved oxygen (DO), biochemical oxygen demand (BOD), total hardness, chemical oxygen demand (COD), bicarbonates $\left(\mathrm{HCO}_{3}{ }^{2}\right)$, acidity and alkalinity were calculated by titrimetric analysis, whereas total solids (TS) and total suspended solids (TSS) were obtained by gravimetric method. The parameters were determined by analyzing three replicates of each sample following the standard methods of APHA (2012).

Sample digestion and heavy metal analysis : The samples were digested by nitric acid digestion method as described in APHA (2012). Heavy metals concentration in digested samples was estimated by atomic absorption spectrophotometer (PerkinElmer, Model- Analyst 700) by running three replicates of each sample. For standardization process, the calibration curves were prepared separately for all the metals by running different concentrations of standard solutions. A reagent blank sample was run throughout the analysis as reference.

Statistical analysis : Data obtained was subjected to statistical analysis for mean and standard deviation (SD) using MS Excel 2007 and correlation matrix using SPSS 12.0.

\section{Results and Discussion}

The mean $\pm S D$ values of various physico-chemical parameters of industrial effluents observed during February 2013 to January 2014 are given in Tables 1-4. To assess the pollution load of composite discharged effluents, the observed values of various parameters were compared with the prescribed limits of Bureau of Indian Standards (BIS, 2012).

In the present study, pH of industrial effluents was slightly alkaline (7.89) (Table 2) at Cluster B in the month of June, whereas $\mathrm{pH}$ of the effluents was observed to be quite acidic (5.33) (Table 3) at Cluster C in the month of March. It may be likely due to the use or spill of either highly acidic raw materials or biproducts from the industries under operation dealing with the production of cosmetics, perfumes, soaps, chemicals, aluminum and zinc based alloys and capacitors, which finally find their way to the effluents. The results are in the line with the work of Lokhande et al. (2011) who reported approximately similar pH range (5.2 to 8.7) of the effluents discharged from Taloja industrial area at Mumbai, India. In contrary, Adakole and Abolude (2009) observed considerably broader $\mathrm{pH}$ range (1.70-11.80) of the effluents discharged from industrial units involved in metal finishing works at Zaria, Nigeria. Maximum temperature $\left(31.13^{\circ} \mathrm{C}\right)$ (Table 3 ) of effluents was recorded at Cluster $\mathrm{C}$ in the month of July, while minimum temperature $\left(24.4^{\circ} \mathrm{C}\right.$ ) (Table 4 ) was observed at Cluster $D$ in November. The temperature of effluents observed in the present study were quite lower as compared to the observations of Siyanbola et al. (2011) who reported comparatively higher temperature $\left(35.35^{\circ} \mathrm{C}\right)$ of industrial effluents discharged from Lagos State, Nigeria. Bae et al. (2016) studied the alteration of thermal regimes in the Mediterranean stream in Korea and reported relatively elevated temperature level of stream might have significant behavioral alterations in fish assemblage. 
The maximum values of TS (2843.33 $\left.\mathrm{mg} \mathrm{I}^{-1}\right)$ and TDS (2107.67 $\mathrm{mg} \mathrm{l}^{-1}$ ) (Table 3 ) were observed in the effluents of Cluster $C$ in the month of February and July, respectively, while minimum values of TS (416.67 $\mathrm{mg} \mathrm{l}^{-1}$ ) (Table 3) and TDS (187.33 $\mathrm{mg} \mathrm{l}^{-1}$ ) (Table 4) were recorded in the effluents of Cluster $C$ in the month of July and from that of Cluster D in January, respectively. In a pollution load assessment study at an Industrial complex at Dehradun, India; Singh et al. (2012) reported relatively higher values of TDS (2655.43 $\mathrm{mg} \mathrm{l}^{-1}$ ) in wastewater which are comparable to the present study. In contrast, Lokhande et al. (2011) reported quite lower value for TS (1475.6 mg I') and significantly higher values for TDS $\left(13499.2 \mathrm{mgl}^{-1}\right)$ in the effluents discharged from Taloja industrial area of Mumbai, India. In the study, maximum value of TSS (970.33 $\mathrm{mg} \mathrm{l}^{-1}$ ) (Table 3) was observed in the effluents at Cluster $C$ in the month of April. At the same time, minimum value of TSS (58.67 mg l-1) (Table 3) was recorded in the month of July from the same cluster. Higher TDS in wastewater might be attributed due to frequent use of salts in the manufacturing processes in different industries.
The turbidity in effluents was maximum (118.67 NTU) (Table 4) at Cluster D in the month of March whereas minimum turbidity (1.0 NTU) (Table 3) was recorded at Cluster $\mathrm{C}$ in the month of April. Islam et al. (2011) reported quite similar values of turbidity (130.37 NTU) in textile effluents, which are comparable with the present observations.

The maximum concentration of DO (5.33 $\left.\mathrm{mg} \mathrm{l}^{-1}\right)$ (Table 3) was observed in the effluents discharged from Cluster $C$ in the month of June, while DO was found to be nil at Cluster A (Table 1) in June, July and November at Cluster B (Table 2) in March and September; at Cluster C (Table 3) in August and September and at Cluster D (Table 4). These observations are comparable (nil) with the work of Paul et al. (2012) who characterized textile industry waste water in Solapur, India and observed nil values of DO in the textile effluents. Similar findings of DO (nil) were reported by Siyanbola et al. (2011) at industrial effluent of Lagos State, Nigeria. Anoxic conditions of effluents at SIIDCUL-IE might be possibly due to higher content of organic matter in wastewater

Table 1 : Physico-chemical characteristics of industrial effluent at Cluster-A(CA) at SIIDCUL-IIE, Haridwar during the year 2013-2014

\begin{tabular}{|c|c|c|c|c|c|c|c|c|c|c|c|c|c|}
\hline Parame & Feb & Mar & Apr & May & June & July & Aug & Sep & Oct & Nov & Dec & Jan & BIS \\
\hline $\mathrm{pH}$ & $\begin{array}{l}7.76 \\
\pm 0.37\end{array}$ & $\begin{array}{l}5.17 \\
\pm 0.49\end{array}$ & $\begin{array}{l}7.08 \\
\pm 0.15\end{array}$ & $\begin{array}{l}7.62 \\
\pm 0.21\end{array}$ & $\begin{array}{l}7.02 \\
\pm 0.34\end{array}$ & $\begin{array}{l}6.32 \\
\pm 0.70\end{array}$ & $\begin{array}{l}6.98 \\
\pm 0.89\end{array}$ & $\begin{array}{l}6.54 \\
\pm 0.57\end{array}$ & $\begin{array}{l}6.92 \\
\pm 0.26\end{array}$ & $\begin{array}{l}7.62 \\
\pm 1.47\end{array}$ & $\begin{array}{l}7.43 \\
\pm 0.20\end{array}$ & $\begin{array}{l}7.25 \\
\pm 2.098\end{array}$ & $5.5-9$ \\
\hline Temp & $\begin{array}{l}26.9 \\
\pm 0.38\end{array}$ & $\begin{array}{l}27.5 \\
\pm 0.49\end{array}$ & $\begin{array}{l}28.4 \\
\pm 0.15\end{array}$ & $\begin{array}{l}30.5 \\
\pm 1.21\end{array}$ & $\begin{array}{l}29.5 \\
\pm 0.34\end{array}$ & $\begin{array}{l}29.4 \\
\pm 0.70\end{array}$ & $\begin{array}{l}29.2 \\
\pm 0.89\end{array}$ & $\begin{array}{l}29.6 \\
\pm 0.57\end{array}$ & $\begin{array}{l}28.5 \\
\pm 0.26\end{array}$ & $\begin{array}{l}26.3 \\
\pm 1.47\end{array}$ & $\begin{array}{l}25.7 \\
\pm 0.20\end{array}$ & $\begin{array}{l}27.5 \\
\pm 2.09\end{array}$ & \\
\hline TS & $\begin{array}{l}656.67 \\
\pm 21.79\end{array}$ & $\begin{array}{l}953.33 \\
\pm 20.33\end{array}$ & $\begin{array}{l}706.67 \\
\pm 27.38\end{array}$ & $\begin{array}{l}626.67 \\
\pm 12.22\end{array}$ & $\begin{array}{l}665 \\
\pm 73.65\end{array}$ & $\begin{array}{l}596.67 \\
\pm 70.94\end{array}$ & $\begin{array}{l}587.33 \\
\pm 20.2\end{array}$ & $\begin{array}{l}606.67 \\
\pm 10.17\end{array}$ & $\begin{array}{l}513.33 \\
\pm 94.51\end{array}$ & $\begin{array}{l}533.33 \\
\pm 115.47\end{array}$ & $\begin{array}{l}606.67 \\
\pm 83.26\end{array}$ & $\begin{array}{l}1230 \\
\pm 206.64\end{array}$ & \\
\hline TSS & $\begin{array}{l}173.3 \\
\pm 12.02\end{array}$ & $\begin{array}{l}254 \\
\pm 19.36\end{array}$ & $\begin{array}{l}174.7 \\
\pm 13.4\end{array}$ & $\begin{array}{l}203.7 \\
\pm 18.68\end{array}$ & $\begin{array}{l}159.7 \\
\pm 8.04\end{array}$ & $\begin{array}{l}165.3 \\
\pm 11.4\end{array}$ & $\begin{array}{l}224.7 \\
\pm 16.81\end{array}$ & $\begin{array}{l}101.3 \\
\pm 9.08\end{array}$ & $\begin{array}{l}140.3 \\
\pm 6.24\end{array}$ & $\begin{array}{l}150.3 \\
\pm 3.85\end{array}$ & $\begin{array}{l}172.7 \\
\pm 12.73\end{array}$ & $\begin{array}{l}842.3 \\
\pm 45.93\end{array}$ & \\
\hline TDS & $\begin{array}{l}483.33 \\
\pm 12.03\end{array}$ & $\begin{array}{l}699.33 \\
\pm 43.89\end{array}$ & $\begin{array}{l}532 \\
\pm 47.18\end{array}$ & $\begin{array}{l}423 \\
\pm 25.64\end{array}$ & $\begin{array}{l}417 \\
\pm 27.92\end{array}$ & $\begin{array}{l}431.33 \\
\pm 24.28\end{array}$ & $\begin{array}{l}362.67 \\
\pm 28.83\end{array}$ & $\begin{array}{l}505.33 \\
\pm 41.18\end{array}$ & $\begin{array}{l}379.67 \\
\pm 38.22\end{array}$ & $\begin{array}{l}383 \\
\pm 14.95\end{array}$ & $\begin{array}{l}437.33 \\
\pm 32.85\end{array}$ & $\begin{array}{l}621 \\
\pm 40.47\end{array}$ & 500 \\
\hline Turbidity & $\begin{array}{l}8.33 \\
\pm 0.12\end{array}$ & $\begin{array}{l}34.3 \\
\pm 1.56\end{array}$ & $\begin{array}{l}4.67 \\
\pm 0.51\end{array}$ & $\begin{array}{l}6.0 \\
\pm 0.35\end{array}$ & $\begin{array}{l}5.23 \\
\pm 0.15\end{array}$ & $\begin{array}{l}1.33 \\
\pm 0.07\end{array}$ & $\begin{array}{l}4.83 \\
\pm 0.30\end{array}$ & $\begin{array}{l}4.07 \\
\pm 0.99\end{array}$ & $\begin{array}{l}8.23 \\
\pm 0.14\end{array}$ & $\begin{array}{l}2.27 \\
\pm 0.48\end{array}$ & $\begin{array}{l}3.23 \\
\pm 0.30\end{array}$ & $\begin{array}{l}9.67 \\
\pm .07\end{array}$ & \\
\hline DO & $\begin{array}{l}0.23 \\
\pm 0\end{array}$ & $\begin{array}{l}1.63 \\
\pm 0.07\end{array}$ & $\begin{array}{l}1.20 \\
\pm 0.08\end{array}$ & $\begin{array}{l}1.13 \\
\pm 0.96\end{array}$ & Nil & $\begin{array}{l}0.37 \\
\pm 0.04\end{array}$ & Nil & $\begin{array}{l}0.7 \\
\pm 0.01\end{array}$ & $\begin{array}{l}0.37 \\
\pm 0.04\end{array}$ & Nil & $\begin{array}{l}1.33 \\
\pm 0.15\end{array}$ & $\begin{array}{l}0.17 \\
\pm 0.04\end{array}$ & \\
\hline BOD & $\begin{array}{l}57 \\
\pm 3.32\end{array}$ & $\begin{array}{l}176.67 \\
\pm 11.45\end{array}$ & $\begin{array}{l}147.60 \\
\pm 4.55\end{array}$ & $\begin{array}{l}110 \\
\pm 5.28\end{array}$ & $\begin{array}{l}167.37 \\
\pm 12.20\end{array}$ & $\begin{array}{l}226.67 \\
\pm 21.17\end{array}$ & $\begin{array}{l}90 \\
\pm 8.2\end{array}$ & $\begin{array}{l}220.67 \\
\pm 11.3\end{array}$ & $\begin{array}{l}283.33 \\
\pm 18.5\end{array}$ & $\begin{array}{l}106.67 \\
\pm 8.55\end{array}$ & $\begin{array}{l}223.33 \\
\pm 15.81\end{array}$ & $\begin{array}{l}276.67 \\
\pm 16.22\end{array}$ & 30 \\
\hline COD & $\begin{array}{l}760 \\
\pm 21.32\end{array}$ & $\begin{array}{l}606.67 \\
\pm 12.91\end{array}$ & $\begin{array}{l}166.67 \\
\pm 12.2\end{array}$ & $\begin{array}{l}200 \\
\pm 9.20\end{array}$ & $\begin{array}{l}293.33 \\
\pm 6.26\end{array}$ & $\begin{array}{l}241.67 \\
\pm 13.74\end{array}$ & $\begin{array}{l}193.33 \\
\pm 5.57\end{array}$ & $\begin{array}{l}262.67 \\
\pm 15.32\end{array}$ & $\begin{array}{l}353.33 \\
\pm 10.51\end{array}$ & $\begin{array}{l}213.33 \\
\pm 183.27\end{array}$ & $\begin{array}{l}400 \\
\pm 17.21\end{array}$ & $\begin{array}{l}533.33 \\
\pm 24.38\end{array}$ & 250 \\
\hline $\mathrm{HCO}_{3}^{2-}$ & $\begin{array}{l}410.73 \\
\pm 27.61\end{array}$ & $\begin{array}{l}376.17 \\
\pm 4.65\end{array}$ & $\begin{array}{l}246.03 \\
\pm 12.38\end{array}$ & $\begin{array}{l}538.83 \\
\pm 0.18\end{array}$ & $\begin{array}{l}296.87 \\
\pm 14.61\end{array}$ & $\begin{array}{l}162.67 \\
\pm 8.09\end{array}$ & $\begin{array}{l}203.33 \\
\pm 16.57\end{array}$ & $\begin{array}{l}248.07 \\
\pm 3.79\end{array}$ & $\begin{array}{l}205.37 \\
\pm 17.38\end{array}$ & $\begin{array}{l}276.53 \\
\pm 9.22\end{array}$ & $\begin{array}{l}227.73 \\
\pm 8.74\end{array}$ & $\begin{array}{l}286.7 \\
\pm 17.9\end{array}$ & \\
\hline Hardness & $\begin{array}{l}373.33 \\
\pm 3.27\end{array}$ & $\begin{array}{l}520 \\
\pm 11.43\end{array}$ & $\begin{array}{l}506.67 \\
\pm 4.19\end{array}$ & $\begin{array}{l}546.67 \\
\pm 11.10\end{array}$ & $\begin{array}{l}506.67 \\
\pm 23.09\end{array}$ & $\begin{array}{l}573.33 \\
\pm 17.31\end{array}$ & $\begin{array}{l}480 \\
\pm 0\end{array}$ & $\begin{array}{l}488.67 \\
\pm 15.45\end{array}$ & $\begin{array}{l}693.33 \\
\pm 22.2\end{array}$ & $\begin{array}{l}653.3 \\
\pm 23.09\end{array}$ & $\begin{array}{l}653.33 \\
\pm 21.11\end{array}$ & $\begin{array}{l}640 \\
\pm 40.0\end{array}$ & \\
\hline Acidity & $\begin{array}{l}92.5 \\
\pm 1.36\end{array}$ & $\begin{array}{l}70.83 \\
\pm 6.08\end{array}$ & $\begin{array}{l}101.7 \\
\pm 7.65\end{array}$ & $\begin{array}{l}96.67 \\
\pm 8.33\end{array}$ & $\begin{array}{l}66.67 \\
\pm 16.02\end{array}$ & $\begin{array}{l}100 \\
\pm 8.66\end{array}$ & $\begin{array}{l}71.67 \\
\pm 2.80\end{array}$ & $\begin{array}{l}82.33 \\
\pm 6.26\end{array}$ & $\begin{array}{l}69.17 \\
\pm 3.46\end{array}$ & $\begin{array}{l}96.67 \\
\pm 20.33\end{array}$ & $\begin{array}{l}23.33 \\
\pm 1.46\end{array}$ & $\begin{array}{l}83.33 \\
\pm 1.76\end{array}$ & \\
\hline Alkalinity & $\begin{array}{l}466.7 \\
\pm 28.87\end{array}$ & $\begin{array}{l}500 \\
\pm 47.22\end{array}$ & $\begin{array}{l}506.7 \\
\pm 6.19\end{array}$ & $\begin{array}{l}650 \\
\pm 70.11\end{array}$ & $\begin{array}{l}583.3 \\
\pm 28.87\end{array}$ & $\begin{array}{l}466.7 \\
\pm 27.74\end{array}$ & $\begin{array}{l}566.7 \\
\pm 17.74\end{array}$ & $\begin{array}{l}558.3 \\
\pm 7.63\end{array}$ & $\begin{array}{l}550 \\
\pm 20\end{array}$ & $\begin{array}{l}533.3 \\
\pm 28.9\end{array}$ & $\begin{array}{l}633.3 \\
\pm 2.00\end{array}$ & $\begin{array}{l}600 \\
\pm 50.0\end{array}$ & \\
\hline K & $\begin{array}{l}2.27 \\
\pm 0.13\end{array}$ & $\begin{array}{l}7.97 \\
\pm 0.66\end{array}$ & $\begin{array}{l}4.27 \\
\pm 0.10\end{array}$ & $\begin{array}{l}4.4 \\
\pm 0.26\end{array}$ & $\begin{array}{l}3.43 \\
\pm 0.25\end{array}$ & $\begin{array}{l}3.03 \\
\pm 0.02\end{array}$ & $\begin{array}{l}3.1 \\
\pm 0.25\end{array}$ & $\begin{array}{l}3.2 \\
\pm 0\end{array}$ & $\begin{array}{l}12.0 \\
\pm 0.27\end{array}$ & $\begin{array}{l}3.4 \\
\pm 0.44\end{array}$ & $\begin{array}{l}3.0 \\
\pm 0.0\end{array}$ & $\begin{array}{l}5.27 \\
\pm 0.29\end{array}$ & \\
\hline$P$ & $\begin{array}{l}1.17 \\
\pm 0.04\end{array}$ & $\begin{array}{l}1.17 \\
\pm 0.01\end{array}$ & $\begin{array}{l}0.83 \\
\pm 0.08\end{array}$ & $\begin{array}{l}0.83 \\
\pm 0.06\end{array}$ & $\begin{array}{l}1.67 \\
\pm 0.09\end{array}$ & $\begin{array}{l}0.33 \\
\pm 0.09\end{array}$ & $\begin{array}{l}2.73 \\
\pm 0.16\end{array}$ & $\begin{array}{l}2.17 \\
\pm 0.17\end{array}$ & $\begin{array}{l}4.5 \\
\pm 0.82\end{array}$ & $\begin{array}{l}1.17 \\
\pm 0.25\end{array}$ & $\begin{array}{l}4.73 \\
\pm 0.19\end{array}$ & $\begin{array}{l}5.33 \\
\pm 0.21\end{array}$ & 5 \\
\hline
\end{tabular}

All parameters are expressed in $\mathrm{mg} \mathrm{l}^{-1}$ except $\mathrm{pH}$, temperature $\left({ }^{\circ} \mathrm{C}\right)$ and turbidity (NTU); BIS: Bureau of Indian Standards for inland disposal of effluents; Valuse are mean \pm SD 
discharged from the industries, which on decomposition, depletes oxygen content in it. The maximum value of BOD (453.33 $\mathrm{mg} \mathrm{I}^{1}$ ) (Table 3 ) in the effluents was recorded from Cluster $\mathrm{C}$ in the month of January, while minimum value of BOD ( $\left.57 \mathrm{mg} \mathrm{l}^{-1}\right)$ (Table 1) was observed in the effluents from Cluster $A$ in the month of February. Singare and Dhabarde (2014) reported BOD values $\left(546 \mathrm{mg} \mathrm{l}^{-1}\right)$ in pharmaceutical industry effluents at Mumbai, which were significantly higher than that reported in the present study. The values of $B O D$ observed in the present study were quite lower than that reported by Haydar et al. (2016) (BOD $2400 \mathrm{mg} \mathrm{l}^{-1}$ ) and Surti (2016)BOD (744 $\left.\mathrm{mgl}^{-1}\right)$, respectively.

Elevated BOD levels in the industrial effluents indicated the presence of larger quantities of organic matter i.e. biologically degradable, thereby consuming DO and possibly resulting up to its depletion level. If BOD level is too high, water could be at risk for further contamination, interfering with the treatment process and affecting the end product (Singh et al., 1998). At SIIDCUL-IE, the maximum value of COD (1003.3 $\mathrm{mg} \mathrm{l}^{-1}$ ) (Table 3 ) in effluents was observed at Cluster $C$ in the month of January, while minimum
COD (125.33 $\mathrm{mgl}^{-1}$ ) (Table 3) was recorded at the same Cluster in July. A relatively higher observation for COD $\left(1254.50 \mathrm{mg} \mathrm{l}^{-1}\right)$ was reported by Siyanbola et al. (2011) in the effluents of different industries at Lagos Estate, Nigeria. In contrast, Singare and Dhabarde (2014) reported considerably high COD values (1271 $\left.\mathrm{mgl}^{-1}\right)$ in a study at Dombivali Industrial Belt of Mumbai, India.

The values of $B O D$ and $C O D$ in the effluents from Cluster $C$ were observed to be much higher (453.33 $\mathrm{mg} \mathrm{l}^{-1}$ and $1003.3 \mathrm{mgl}^{-1}$ ) than the permissible limits of BOD (30 $\left.\mathrm{mg} \mathrm{l}^{-1}\right)$ and $\operatorname{COD~}\left(250 \mathrm{mg} \mathrm{l}^{-1}\right)$ as permissible BIS (1991). It might possibly be due to the fact that this Cluster has numerous industrial units that formulate and manufacture drugs and pharma-products, thereby contributing to high organic load. A similar trend for the values of BOD and COD values beyond the permissible limits was reported by Singare and Dhabarde (2014) for BOD (546 $\left.\mathrm{mgl}^{-1}\right)$ and COD (1045 $\left.\mathrm{mg} \mathrm{l}^{-1}\right)$ in the effluent of pharmaceutical industries at Mumbai, India.

The maximum concentration of bicarbonates (924.53 $\mathrm{mg}^{-1}$ ) (Table 2) in the effluents was recorded at Cluster B in the

Table 2 : Physico-chemical characteristics of industrial effluent at Cluster-B (CB) at SIIDCUL-IIE, Haridwar during the year 2013-2014

\begin{tabular}{|c|c|c|c|c|c|c|c|c|c|c|c|c|c|}
\hline Parameter & r Feb & Mar & Apr & May & June & July & Aug & Sep & Oct & Nov & Dec & Jan & BIS \\
\hline $\mathrm{pH}$ & $\begin{array}{l}7.77 \\
\pm 0.45\end{array}$ & $\begin{array}{l}6.07 \\
\pm 0.38\end{array}$ & $\begin{array}{l}7.52 \\
\pm 0.75\end{array}$ & $\begin{array}{l}7.6 \\
\pm 0.11\end{array}$ & $\begin{array}{l}7.89 \\
\pm 0.58\end{array}$ & $\begin{array}{l}6.95 \\
\pm 0.53\end{array}$ & $\begin{array}{l}6.68 \\
\pm 0.26\end{array}$ & $\begin{array}{l}6.67 \\
\pm 0.15\end{array}$ & $\begin{array}{l}7.08 \\
\pm 0.35\end{array}$ & $\begin{array}{l}7.49 \\
\pm 0.37\end{array}$ & $\begin{array}{l}7.55 \\
\pm 0.14\end{array}$ & $\begin{array}{l}7.55 \\
\pm 0.63\end{array}$ & 5.5-9 \\
\hline Temp & $\begin{array}{l}25.6 \\
\pm 0.45\end{array}$ & $\begin{array}{l}26.5 \\
\pm 0.38\end{array}$ & $\begin{array}{l}27.9 \\
\pm 0.75\end{array}$ & $\begin{array}{l}30.1 \\
\pm 1.11\end{array}$ & $\begin{array}{l}29.4 \\
\pm 0.58\end{array}$ & $\begin{array}{l}30.1 \\
\pm 0.93\end{array}$ & $\begin{array}{l}29.2 \\
\pm 1.06\end{array}$ & $\begin{array}{l}29.9 \\
\pm 0.15\end{array}$ & $\begin{array}{l}29.43 \\
\pm 0.35\end{array}$ & $\begin{array}{l}27.93 \\
\pm 0.38\end{array}$ & $\begin{array}{l}27.57 \\
\pm 1.84\end{array}$ & $\begin{array}{l}26.9 \\
\pm 1.84\end{array}$ & \\
\hline TS & $\begin{array}{l}756.67 \\
\pm 0.79\end{array}$ & $\begin{array}{l}1753.3 \\
\pm 74.4\end{array}$ & $\begin{array}{l}613.33 \\
\pm 25.95\end{array}$ & $\begin{array}{l}766.67 \\
\pm 30.6\end{array}$ & $\begin{array}{l}686.67 \\
\pm 13.72\end{array}$ & $\begin{array}{l}473.33 \\
\pm 30.551\end{array}$ & $\begin{array}{l}580 \\
\pm 34.97\end{array}$ & $\begin{array}{l}737.33 \\
\pm 21.44\end{array}$ & $\begin{array}{l}526.67 \\
\pm 23.267\end{array}$ & $\begin{array}{l}833.33 \\
\pm 51.66\end{array}$ & $\begin{array}{l}900 \\
\pm 45.89\end{array}$ & $\begin{array}{l}566.67 \\
\pm 52.75\end{array}$ & \\
\hline TSS & $\begin{array}{l}195 \\
\pm 14.6\end{array}$ & $\begin{array}{l}955.33 \\
\pm 50.4\end{array}$ & $\begin{array}{l}61.333 \\
\pm 7.01\end{array}$ & $\begin{array}{l}1267 \\
\pm 18.0\end{array}$ & $\begin{array}{l}185.67 \\
\pm 17.7\end{array}$ & $\begin{array}{l}64.667 \\
\pm 1.69\end{array}$ & $\begin{array}{l}272.33 \\
\pm 24.0\end{array}$ & $\begin{array}{l}116.67 \\
\pm 11.02\end{array}$ & $\begin{array}{l}70.33 \\
\pm 2.06\end{array}$ & $\begin{array}{l}2038 \\
\pm 30.10\end{array}$ & $\begin{array}{l}392.67 \\
\pm 35.4\end{array}$ & $\begin{array}{l}191 \\
\pm 7.70\end{array}$ & 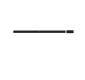 \\
\hline TDS & $\begin{array}{l}561.6 \\
\pm 16.18\end{array}$ & $\begin{array}{l}798 \\
\pm 28.53\end{array}$ & $\begin{array}{l}552 \\
\pm 21.19\end{array}$ & $\begin{array}{l}500 \\
\pm 126.30\end{array}$ & $\begin{array}{l}504.33 \\
\pm 126.2\end{array}$ & $\begin{array}{r}408.67 \\
0 \pm 29.95\end{array}$ & $\begin{array}{l}307.67 \\
\pm 118.6\end{array}$ & $\begin{array}{l}620.67 \\
\pm 98.42\end{array}$ & $\begin{array}{l}415 \\
\pm 36.71\end{array}$ & $\begin{array}{l}462 \\
\pm 120.86\end{array}$ & $\begin{array}{l}506 \\
\pm 84.33\end{array}$ & $\begin{array}{l}375.67 \\
\pm 82.88\end{array}$ & 500 \\
\hline Turbidity & $\begin{array}{l}4.67 \\
\pm 2.89\end{array}$ & $\begin{array}{l}54.3 \\
\pm 60.6\end{array}$ & $\begin{array}{l}1.0 \\
\pm 0\end{array}$ & $\begin{array}{l}14.7 \\
\pm 1.4\end{array}$ & $\begin{array}{l}5.9 \\
\pm 0.1\end{array}$ & $\begin{array}{l}5.33 \\
\pm 0.46\end{array}$ & $\begin{array}{l}6.07 \\
\pm 0.69\end{array}$ & $\begin{array}{l}7.3 \\
\pm 0.12\end{array}$ & $\begin{array}{l}4.23 \\
\pm 0.23\end{array}$ & $\begin{array}{l}5.67 \\
\pm 0.25\end{array}$ & $\begin{array}{l}9.8 \\
\pm 0.86\end{array}$ & $\begin{array}{l}7.7 \\
\pm 0.20\end{array}$ & \\
\hline DO & $\begin{array}{l}2.37 \\
\pm 0.09\end{array}$ & Nil & $\begin{array}{l}4.13 \\
\pm 0.10\end{array}$ & $\begin{array}{l}4.07 \\
\pm 0.20\end{array}$ & $\begin{array}{l}4.3 \\
\pm 0.13\end{array}$ & $\begin{array}{l}3.3 \\
\pm 0.16\end{array}$ & $\begin{array}{l}1.0 \\
\pm 0.03\end{array}$ & Nil & $\begin{array}{l}1.87 \\
\pm 0.10\end{array}$ & $\begin{array}{l}0.47 \\
\pm 0.04\end{array}$ & $\begin{array}{l}2.03 \\
\pm 0.15\end{array}$ & $\begin{array}{l}1.67 \\
\pm 0.05\end{array}$ & \\
\hline BOD & $\begin{array}{l}67 \\
\pm 0.38\end{array}$ & $\begin{array}{l}376.67 \\
\pm 26.6\end{array}$ & $\begin{array}{l}108 \\
\pm 0.91\end{array}$ & $\begin{array}{l}146.67 \\
\pm 11.90\end{array}$ & $\begin{array}{l}216.67 \\
\pm 14.70\end{array}$ & $\begin{array}{l}173.33 \\
\pm 7.26\end{array}$ & $\begin{array}{l}166.67 \\
\pm 14.20\end{array}$ & $\begin{array}{l}250 \\
\pm 16.30\end{array}$ & $\begin{array}{l}206.67 \\
\pm 15.27\end{array}$ & $\begin{array}{l}91.667 \\
\pm 3.29\end{array}$ & $\begin{array}{l}100.83 \\
\pm 8.75\end{array}$ & $\begin{array}{l}173.33 \\
\pm 7.94\end{array}$ & 30 \\
\hline COD & $\begin{array}{l}506.67 \\
\pm 15.1\end{array}$ & $\begin{array}{l}466.67 \\
\pm 20.30\end{array}$ & $\begin{array}{r}186.67 \\
\pm 10.51\end{array}$ & $\begin{array}{l}186.67 \\
\pm 11.10\end{array}$ & $\begin{array}{l}213.33 \\
\pm 8.26\end{array}$ & $\begin{array}{l}282 \\
\pm 1.85\end{array}$ & $\begin{array}{l}293.33 \\
\pm 19.24\end{array}$ & $\begin{array}{l}380 \\
\pm 15.97\end{array}$ & $\begin{array}{l}256 \\
\pm 12.48\end{array}$ & $\begin{array}{l}190 \\
\pm 6.60\end{array}$ & $\begin{array}{l}176 \\
\pm 7.54\end{array}$ & $\begin{array}{l}346.67 \\
\pm 19.03\end{array}$ & 250 \\
\hline $\mathrm{HCO}_{3}{ }^{2-}$ & $\begin{array}{l}372.1 \\
\pm 29.3\end{array}$ & $\begin{array}{l}406.67 \\
\pm 5.22\end{array}$ & $\begin{array}{l}231.8 \\
\pm 9.93\end{array}$ & $\begin{array}{l}396.5 \\
\pm 12.05\end{array}$ & $\begin{array}{l}412.77 \\
\pm 19.90\end{array}$ & $\begin{array}{l}357.87 \\
\pm 24.11\end{array}$ & $\begin{array}{l}176.9 \\
\pm 8.74\end{array}$ & $\begin{array}{l}276.23 \\
\pm 10.95\end{array}$ & $\begin{array}{l}217.57 \\
\pm 19.69\end{array}$ & $\begin{array}{l}357.87 \\
\pm 1.52\end{array}$ & $\begin{array}{l}234.67 \\
\pm 21.17\end{array}$ & $\begin{array}{l}924.53 \\
\pm 54.70\end{array}$ & \\
\hline Hardness & $\begin{array}{l}426.67 \\
\pm 18.3\end{array}$ & $\begin{array}{l}373.33 \\
\pm 21.10\end{array}$ & $\begin{array}{l}546.67 \\
\pm 36.19\end{array}$ & $\begin{array}{l}426.67 \\
\pm 13.27\end{array}$ & $\begin{array}{l}600 \\
\pm 43.31\end{array}$ & $\begin{array}{l}480 \\
\pm 15.83\end{array}$ & $\begin{array}{l}466.67 \\
\pm 23.09\end{array}$ & $\begin{array}{l}460.67 \\
\pm 3.05\end{array}$ & $\begin{array}{l}680 \\
\pm 10.0\end{array}$ & $\begin{array}{l}640 \\
\pm 14.36\end{array}$ & $\begin{array}{l}613.33 \\
\pm 22.2\end{array}$ & $\begin{array}{l}666.67 \\
\pm 31.10\end{array}$ & \\
\hline Acidity & $\begin{array}{l}71.67 \\
\pm 7.64\end{array}$ & $\begin{array}{l}96.67 \\
\pm 1.0\end{array}$ & $\begin{array}{l}88.33 \\
\pm 8.40\end{array}$ & $\begin{array}{l}66.67 \\
\pm 3.20\end{array}$ & $\begin{array}{l}70.83 \\
\pm 1.10\end{array}$ & $\begin{array}{l}216.7 \\
\pm 11.66\end{array}$ & $\begin{array}{l}50.83 \\
\pm 2.6\end{array}$ & $\begin{array}{l}62.83 \\
\pm 2.88\end{array}$ & $\begin{array}{l}181.7 \\
\pm 11.0\end{array}$ & $\begin{array}{l}77.5 \\
\pm 7.4\end{array}$ & $\begin{array}{l}71.17 \\
\pm 0.2\end{array}$ & $\begin{array}{l}277.5 \\
\pm 0.34\end{array}$ & \\
\hline Alkalinity & $\begin{array}{l}433.3 \\
\pm 7.38\end{array}$ & $\begin{array}{l}450 \\
\pm 6.66\end{array}$ & $\begin{array}{l}546.7 \\
\pm 22.19\end{array}$ & $\begin{array}{l}600 \\
\pm 50.00\end{array}$ & $\begin{array}{l}573.3 \\
\pm 13.77\end{array}$ & $\begin{array}{l}396.7 \\
\pm 16.55\end{array}$ & $\begin{array}{l}483.3 \\
\pm 28.87\end{array}$ & $\begin{array}{l}546.7 \\
\pm 51.08\end{array}$ & $\begin{array}{l}416.7 \\
\pm 21.71\end{array}$ & $\begin{array}{l}543.3 \\
\pm 30.28\end{array}$ & $\begin{array}{l}566.7 \\
\pm 16.38\end{array}$ & $\begin{array}{l}382.5 \\
\pm 22.2\end{array}$ & \\
\hline K & $\begin{array}{l}4.53 \\
\pm 0.8\end{array}$ & $\begin{array}{l}7.57 \\
\pm 0.76\end{array}$ & $\begin{array}{l}5.5 \\
\pm 0.32\end{array}$ & $\begin{array}{l}3.37 \\
\pm 0.10\end{array}$ & $\begin{array}{l}6.47 \\
\pm 0.40\end{array}$ & $\begin{array}{l}6.03 \\
\pm 0.22\end{array}$ & $\begin{array}{l}6.2 \\
\pm 0.44\end{array}$ & $\begin{array}{l}3.4 \\
\pm 0.02\end{array}$ & $\begin{array}{l}5.0 \\
\pm 0.08\end{array}$ & $\begin{array}{l}3.75 \\
\pm 0.12\end{array}$ & $\begin{array}{l}5.73 \\
\pm 0.4\end{array}$ & $\begin{array}{l}170 \\
\pm 16.00\end{array}$ & \\
\hline$P$ & $\begin{array}{l}0.83 \\
\pm 0.01\end{array}$ & $\begin{array}{l}0.5 \\
\pm 0.0\end{array}$ & $\begin{array}{l}0.33 \\
\pm 0.02\end{array}$ & $\begin{array}{l}0.83 \\
\pm 0.04\end{array}$ & $\begin{array}{l}1.5 \\
\pm 0.08\end{array}$ & $\begin{array}{l}0.21 \\
\pm 0.01\end{array}$ & $\begin{array}{l}2.0 \\
\pm 0.02\end{array}$ & $\begin{array}{l}4.67 \\
\pm 0.31\end{array}$ & $\begin{array}{l}5.0 \\
\pm 0.16\end{array}$ & $\begin{array}{l}5.0 \\
\pm 0.36\end{array}$ & $\begin{array}{l}2.27 \\
\pm 0.05\end{array}$ & $\begin{array}{l}4.27 \\
\pm 0.23\end{array}$ & 5 \\
\hline
\end{tabular}

All parameters are expressed in $\mathrm{mg} \mathrm{I}^{-1}$ except $\mathrm{pH}$, temperature $\left({ }^{\circ} \mathrm{C}\right.$ ) and turbidity (NTU); BIS: Bureau of Indian Standards for inland disposal of effluents; Vlaues are mean \pm SD 
month of January, whereas minimum concentration of bicarbonates (154.5 $\mathrm{mg} \mathrm{l}^{-1}$ ) (Table 3) was observed at Cluster C in the month of July. The concentration of bicarbonates was quite higher than the range values of bicarbonates $\left(390-430 \mathrm{mg} \mathrm{I}^{-1}\right)$ reported by Ahmad et al. (2012) in the effluent of dye industry at Ujjain, India while Smrithi et al. (2012) reported relatively lower bicarbonate content $\left(101.93 \mathrm{mg} \mathrm{l}^{-1}\right)$ in textile mill effluent at Coimbatore, India.

Hardness in SIIDCUL-IE industrial effluents was observed maximum (1613.3 $\mathrm{mg} \mathrm{l}^{-1}$ ) (Table 3 ) at Cluster $\mathrm{C}$ in the month of February, while minimum value of hardness (373.33 $\mathrm{mg} \mathrm{l}^{-1}$ ) (Table 2) was recorded at Cluster B in March. In contrast to these observations, Paul et al. (2012) reported relatively lower value of hardness $\left(1050 \mathrm{mg} \mathrm{l}^{-1}\right)$ in textile mill effluent at Solapur, India. The values (1613.3 $\left.\mathrm{mg} \mathrm{l}^{-1}\right)$ of hardness were considerably higher than the values $\left(800 \mathrm{mg} \mathrm{l}^{-1}\right)$ reported by Yadav and Pathak (2012) in assessment of sugar mill effluent at Khargone (India).
The maximum value of alkalinity $\left(783.33 \mathrm{mg} \mathrm{I}^{-1}\right)$ (Table 4) was observed at Cluster $D$ in February, while minimum value of alkalinity (79.33 $\mathrm{mg} \mathrm{I}^{-1}$ ) (Table 3) was recorded at Cluster $\mathrm{C}$ in September. In contrast, Ahmad et al. (2012) and Paul et al. (2012) reported comparatively lower values of alkalinity in the industrial effluents from dye $\left(430 \mathrm{mg} \mathrm{l}^{-1}\right)$ and textile $\left(500 \mathrm{mg} \mathrm{l}^{-1}\right)$ industries at Ujjain and Solapur cities of India, respectively. The industries involved in production and processing of goods like soap, textile, dying, rubber, tannery, etc., are responsible for alkalinity producing substances in the industrial effluents (Goel, 1997). In SIIDCUL-IE, Cluster $C$ also has abundant functional industries involved in production or processing of the above mentioned products, which might possibly be responsible for relatively more alkaline nature of effluents at this cluster in the month of February.

At SIIDCUL-IE, the maximum value of acidity $\left(277.5 \mathrm{mg} \mathrm{I}^{-1}\right)$ (Table 2) was recorded at Cluster B in the month of January, whereas minimum value of acidity $\left(22.5 \mathrm{mg} \mathrm{l}^{-1}\right)$ (Table 4) was observed at Cluster $D$ in the month of December. These results are

Table 3 : Physico-chemical characteristics of industrial effluent Cluster-C (CC) at SIIDCUL-IIE, Haridwar during the year 2013-2014

\begin{tabular}{|c|c|c|c|c|c|c|c|c|c|c|c|c|c|}
\hline Paramete & $\mathrm{rFeb}$ & Mar & Apr & May & June & July & Aug & Sep & Oct & Nov & Dec & Jan & BIS \\
\hline $\mathrm{pH}$ & $\begin{array}{l}7.08 \\
\pm 0.49\end{array}$ & $\begin{array}{l}5.33 \\
\pm 0.10\end{array}$ & $\begin{array}{l}6.8 \\
\pm 0.21\end{array}$ & $\begin{array}{l}6.5 \\
\pm 0.31\end{array}$ & $\begin{array}{l}5.74 \\
\pm 0.9\end{array}$ & $\begin{array}{l}7.77 \\
\pm 0.65\end{array}$ & $\begin{array}{l}6.71 \\
\pm 0.51\end{array}$ & $\begin{array}{l}6.97 \\
\pm 0.35\end{array}$ & $\begin{array}{l}5.63 \\
\pm 0.47\end{array}$ & $\begin{array}{l}7.33 \\
\pm 0.23\end{array}$ & $\begin{array}{l}6.22 \\
\pm 0.37\end{array}$ & $\begin{array}{l}7.18 \\
\pm 0.18\end{array}$ & $5.5-9$ \\
\hline Temp & $\begin{array}{l}25.37 \\
\pm 0.50\end{array}$ & $\begin{array}{l}25.97 \\
\pm 1.10\end{array}$ & $\begin{array}{l}26.97 \\
\pm 1.21\end{array}$ & $\begin{array}{l}29.57 \\
\pm 0.31\end{array}$ & $\begin{array}{l}28.83 \\
\pm 0.90\end{array}$ & $\begin{array}{l}31.13 \\
\pm 1.05\end{array}$ & $\begin{array}{l}28.77 \\
\pm 0.81\end{array}$ & $\begin{array}{l}28.87 \\
\pm 0.85\end{array}$ & $\begin{array}{l}29.13 \\
\pm 0.47\end{array}$ & $\begin{array}{l}29.7 \\
\pm 3.13\end{array}$ & $\begin{array}{l}26.6 \\
\pm 0.86\end{array}$ & $\begin{array}{l}25.53 \\
\pm 1.18\end{array}$ & \\
\hline TS & $\begin{array}{l}2843.33 \\
\pm 150.33\end{array}$ & $\begin{array}{l}1733.33 \\
\pm 12.83\end{array}$ & $\begin{array}{l}1836.67 \\
\pm 16.53\end{array}$ & $\begin{array}{l}823.33 \\
\pm 16.58\end{array}$ & $\begin{array}{r}1080.0 \\
\pm 81.82\end{array}$ & $\begin{array}{l}416.67 \\
\pm 12.83\end{array}$ & $\begin{array}{l}533.33 \\
\pm 31.47\end{array}$ & $\begin{array}{l}534 \\
\pm 26.22\end{array}$ & $\begin{array}{l}946.667 \\
\pm 480.39\end{array}$ & $\begin{array}{r}683.33 \\
\pm 45.83\end{array}$ & $\begin{array}{l}640 \\
\pm 44.22\end{array}$ & $\begin{array}{l}736.66 \\
\pm 55.07\end{array}$ & \\
\hline TSS & $\begin{array}{l}402.33 \\
\pm 37.33\end{array}$ & $\begin{array}{l}589.67 \\
\pm 38.42\end{array}$ & $\begin{array}{l}970.33 \\
\pm 13.17\end{array}$ & $\begin{array}{l}140 \\
\pm 12.347\end{array}$ & $\begin{array}{l}111.67 \\
7 \pm 7.24\end{array}$ & $\begin{array}{l}58.67 \\
\pm 5.77\end{array}$ & $\begin{array}{l}119.67 \\
\pm 6.42\end{array}$ & $\begin{array}{l}98.67 \\
\pm 3.37\end{array}$ & $\begin{array}{l}161.67 \\
\pm 9.79\end{array}$ & $\begin{array}{l}247.67 \\
\pm 8.53\end{array}$ & $\begin{array}{l}185 \\
\pm 1.61\end{array}$ & $\begin{array}{l}118 \\
\pm 2.58\end{array}$ & \\
\hline TDS & $\begin{array}{l}2107.67 \\
\pm 136.71\end{array}$ & $\begin{array}{l}1145 \\
\pm 83.94\end{array}$ & $\begin{array}{l}866.33 \\
\pm 72.21\end{array}$ & $\begin{array}{l}683.33 \\
\pm 34.12\end{array}$ & $\begin{array}{l}968.33 \\
\pm 19.55\end{array}$ & $\begin{array}{l}358 \\
\pm 19.35\end{array}$ & $\begin{array}{l}413.67 \\
\pm 28.22\end{array}$ & $\begin{array}{l}435.33 \\
\pm 1.57\end{array}$ & $\begin{array}{l}695 \\
\pm 6.62\end{array}$ & $\begin{array}{l}461.6 \\
\pm 7.31\end{array}$ & $\begin{array}{l}455 \\
\pm 11.48\end{array}$ & $\begin{array}{l}618.6 \\
\pm 14.37\end{array}$ & 500 \\
\hline Turbidity & $\begin{array}{l}8.0 \\
\pm 0.89\end{array}$ & $\begin{array}{l}85.33 \\
\pm 6.52\end{array}$ & $\begin{array}{l}14.67 \\
\pm 1.95\end{array}$ & $\begin{array}{l}18.67 \\
\pm 0.01\end{array}$ & $\begin{array}{l}10.67 \\
\pm 0.50\end{array}$ & $\begin{array}{l}3.67 \\
\pm 0.155\end{array}$ & $\begin{array}{l}5.37 \\
\pm 0.47\end{array}$ & $\begin{array}{l}6.07 \\
\pm 0.92\end{array}$ & $\begin{array}{l}47.7 \\
\pm 3.52\end{array}$ & $\begin{array}{l}7.6 \\
\pm 0.07\end{array}$ & $\begin{array}{l}3.53 \\
\pm 0.69\end{array}$ & $\begin{array}{l}54.2 \\
\pm 2.52\end{array}$ & \\
\hline DO & $\begin{array}{l}1.27 \\
\pm 0.04\end{array}$ & $\begin{array}{l}0.93 \\
\pm 0.06\end{array}$ & $\begin{array}{l}3.5 \\
\pm 0.20\end{array}$ & $\begin{array}{l}3.63 \\
\pm 0.55\end{array}$ & $\begin{array}{l}5.33 \\
\pm 0.15\end{array}$ & $\begin{array}{l}3.3 \\
\pm 1.06\end{array}$ & Nil & Nil & $\begin{array}{l}2.0 \\
\pm 0.46\end{array}$ & $\begin{array}{l}1.967 \\
\pm 0.05\end{array}$ & $\begin{array}{l}0.1 \\
\pm 0.07\end{array}$ & Nil & \\
\hline BOD & $\begin{array}{l}143.67 \\
\pm 8.85\end{array}$ & $\begin{array}{l}276.67 \\
\pm 15.72\end{array}$ & $\begin{array}{l}188.83 \\
\pm 6.89\end{array}$ & $\begin{array}{l}210 \\
\pm 20.17\end{array}$ & $\begin{array}{l}145.7 \\
\pm 14.14\end{array}$ & $\begin{array}{l}243.33 \\
\pm 19.70\end{array}$ & $\begin{array}{l}309.33 \\
\pm 26.58\end{array}$ & $\begin{array}{l}318.67 \\
\pm 27.75\end{array}$ & $\begin{array}{l}155 \\
\pm 12.78\end{array}$ & $\begin{array}{l}153.33 \\
\pm 12.02\end{array}$ & $\begin{array}{l}193.33 \\
\pm 18.46\end{array}$ & $\begin{array}{l}453.33 \\
\pm 5.11\end{array}$ & 30 \\
\hline COD & $\begin{array}{l}493.33 \\
\pm 12.22\end{array}$ & $\begin{array}{l}436.67 \\
\pm 36.92\end{array}$ & $\begin{array}{l}293.33 \\
\pm 12.66\end{array}$ & $\begin{array}{l}226.6 \\
\pm 21.44\end{array}$ & $\begin{array}{l}313.33 \\
\pm 5.73\end{array}$ & $\begin{array}{l}125.33 \\
\pm 12.32\end{array}$ & $\begin{array}{l}257.67 \\
\pm 15.13\end{array}$ & $\begin{array}{l}206.67 \\
\pm 18.88\end{array}$ & $\begin{array}{l}176.67 \\
\pm 14.72\end{array}$ & $\begin{array}{l}270 \\
\pm 21.24\end{array}$ & $\begin{array}{l}340 \\
\pm 8.75\end{array}$ & $\begin{array}{l}1003.3 \\
\pm 46.27\end{array}$ & 250 \\
\hline $\mathrm{HCO}_{3}^{-}$ & $\begin{array}{l}254.5 \\
\pm 13.40\end{array}$ & $\begin{array}{l}323.3 \\
\pm 23.86\end{array}$ & $\begin{array}{l}266.4 \\
\pm 14.96\end{array}$ & $\begin{array}{l}376.2 \\
\pm 33.1\end{array}$ & $\begin{array}{l}380.2 \\
\pm 15.2\end{array}$ & $\begin{array}{l}154.5 \\
\pm 9.31\end{array}$ & $\begin{array}{l}205.4 \\
\pm 6.10\end{array}$ & $\begin{array}{l}203.4 \\
\pm 8.11\end{array}$ & $\begin{array}{l}353.8 \\
\pm 19.3\end{array}$ & $\begin{array}{l}254.2 \\
\pm 17.1\end{array}$ & $\begin{array}{l}225.7 \\
\pm 23.99\end{array}$ & $\begin{array}{l}235.9 \\
\pm 19.37\end{array}$ & \\
\hline Hardness & $\begin{array}{l}1613.3 \\
\pm 124.04\end{array}$ & $\begin{array}{r}853.33 \\
+ \pm 72.65\end{array}$ & $\begin{array}{l}506.67 \\
\pm 43.26\end{array}$ & $\begin{array}{l}613.33 \\
\pm 24.39\end{array}$ & $\begin{array}{l}426.67 \\
\pm 23.09\end{array}$ & $\begin{array}{l}493.33 \\
\pm 28.82\end{array}$ & $\begin{array}{l}453.33 \\
\pm 13.09\end{array}$ & $\begin{array}{l}483.33 \\
\pm 45.95\end{array}$ & $\begin{array}{l}560 \\
\pm 8.56\end{array}$ & $\begin{array}{l}626.67 \\
\pm 46.18\end{array}$ & $\begin{array}{l}480 \\
\pm 6.28\end{array}$ & $\begin{array}{l}513.33 \\
\pm 30.82\end{array}$ & \\
\hline Acidity & $\begin{array}{l}110 \\
\pm 8.63\end{array}$ & $\begin{array}{l}120 \\
\pm 4.22\end{array}$ & $\begin{array}{l}99.9 \\
\pm 1.50\end{array}$ & $\begin{array}{l}99.17 \\
\pm 5.92\end{array}$ & $\begin{array}{l}80.73 \\
\pm 6.00\end{array}$ & $\begin{array}{l}120.8 \\
\pm 7.21\end{array}$ & $\begin{array}{l}84.17 \\
\pm 10.65\end{array}$ & $\begin{array}{l}79.33 \\
\pm 7.23\end{array}$ & $\begin{array}{l}173.3 \\
\pm 9.09\end{array}$ & $\begin{array}{l}71.67 \\
\pm 2.7\end{array}$ & $\begin{array}{l}94.17 \\
\pm 3.08\end{array}$ & $\begin{array}{l}142.5 \\
\pm 7.30\end{array}$ & \\
\hline Alkalinity & $\begin{array}{l}416.67 \\
\pm 14.34\end{array}$ & $\begin{array}{l}450.11 \\
\pm 30.90\end{array}$ & $\begin{array}{l}506.67 \\
\pm 13.26\end{array}$ & $\begin{array}{l}483.33 \\
\pm 15.66\end{array}$ & $\begin{array}{l}580 \\
\pm 21.24\end{array}$ & $\begin{array}{l}433.33 \\
\pm 5.83\end{array}$ & $\begin{array}{l}666.67 \\
\pm 3.37\end{array}$ & $\begin{array}{l}79.33 \\
\pm 1.74\end{array}$ & $\begin{array}{l}333.33 \\
\pm 25.38\end{array}$ & $\begin{array}{l}433.33 \\
\pm 25.59\end{array}$ & $\begin{array}{l}433.33 \\
\pm 33.17\end{array}$ & $\begin{array}{l}610 \\
\pm 38.09\end{array}$ & \\
\hline K & $\begin{array}{l}6.53 \\
\pm 0.30\end{array}$ & $\begin{array}{l}7.43 \\
\pm 0.07\end{array}$ & $\begin{array}{l}6.09 \\
\pm 0.39\end{array}$ & $\begin{array}{l}7.13 \\
\pm 0.17\end{array}$ & $\begin{array}{l}2.19 \\
\pm 0.14\end{array}$ & $\begin{array}{l}3.38 \\
\pm 0.23\end{array}$ & $\begin{array}{l}6.97 \\
\pm 0.58\end{array}$ & $\begin{array}{l}2.77 \\
\pm 0.06\end{array}$ & $\begin{array}{l}8.16 \\
\pm 0.19\end{array}$ & $\begin{array}{l}5.20 \\
\pm 0.55\end{array}$ & $\begin{array}{l}5.06 \\
\pm 0.58\end{array}$ & $\begin{array}{l}15.1 \\
\pm 1.10\end{array}$ & \\
\hline$P$ & $\begin{array}{l}0.67 \\
\pm 0.09\end{array}$ & $\begin{array}{l}0.67 \\
\pm 0.08\end{array}$ & $\begin{array}{l}1.83 \\
\pm 0.16\end{array}$ & $\begin{array}{l}1.0 \\
\pm 0.02\end{array}$ & $\begin{array}{l}2.67 \\
\pm 0.05\end{array}$ & $\begin{array}{l}0.17 \\
\pm 0.09\end{array}$ & $\begin{array}{l}3.33 \\
\pm 0.77\end{array}$ & $\begin{array}{l}3.67 \\
\pm 0.29\end{array}$ & $\begin{array}{l}15 \\
\pm 1.80\end{array}$ & $\begin{array}{l}2.83 \\
\pm 0.25\end{array}$ & $\begin{array}{l}3.7 \\
\pm 0.25\end{array}$ & $\begin{array}{l}5.53 \\
\pm 0.02\end{array}$ & 5 \\
\hline
\end{tabular}

All parameters are expressed in $\mathrm{mg} / \mathrm{l}$ except $\mathrm{pH}$, temperature $\left({ }^{\circ} \mathrm{C}\right.$ ) and turbidity (NTU); BIS: Bureau of Indian Standards for inland disposal of effluents; Vlaues are mean \pm SD

Journal of Environmental Biology, March 2017 
in line with the work of Jamaluddin and Nizamuddin (2012) who reported almost similar results for acidity $\left(296.8 \mathrm{mg} \mathrm{l}^{-1}\right)$ in textile mill effluent at Chittagong, Bangladesh.

The maximum value of potassium (25 $\mathrm{mg} \mathrm{l}^{-1}$ ) (Table 4) was observed at Cluster $D$ in the month of May, whereas minimum value $\left(2.19 \mathrm{mg} \mathrm{l}^{-1}\right)$ (Table 3 ) was observed at Cluster $C$ in June. In contrast to these observations, Al-Farraj et al. (2013) reported extremely low concentration of potassium $\left(1.3 \mathrm{mg} \mathrm{l}^{-1}\right)$ in industrial wastewater at Riyadh city, Saudi Arabia.

The maximum value of phosphorus (15.5 $\mathrm{mgl}^{-1}$ ) (Table 4) was recorded at Cluster $D$ in the month of May, whereas phosphorus was observed to be minimum $\left(0.17 \mathrm{mgl}^{-1}\right)$ (Table 3 ) at Cluster $\mathrm{C}$ in the month of July. These results were considerably higher as compared with the findings of Abraha et al. (2014) who reported lower values of phosphorus $\left(3.31 \mathrm{mg} \mathrm{l}^{-1}\right)$ in an assessment study of textile industry effluents at Trigram, Northern Ethiopia. The present values of phosphorus $\left(15.5 \mathrm{mg} \mathrm{l}^{-1}\right)$ were much higher than the permissible limits (phosphorus as dissolved phosphates) $\left(5.0 \mathrm{mg} \mathrm{I}^{-1}\right)$ as prescribed by BIS. Cluster $\mathrm{D}$ had one of the major industrial units involved in manufacturing of homeopathic drugs. Mathie et al. (2007) reported that phosphorus is frequently used as a raw material in formulation of homeopathic remedies. Beside this, boilers are commonly installed in industries to meet their de-mineralized water requirement, where phosphates are extensively used in the treatment of boiler water. Thus, higher concentration of phosphorus in effluents from Cluster $D$ might be likely due to same reasons.

The effects of heavy metals in wastewater vary from beneficial or troublesome to dangerously toxic. Metals like Zn, Fe, $\mathrm{Ca}, \mathrm{Mg}$ are essential for plant growth up to certain level, while $\mathrm{Pb}$, $\mathrm{Cd}, \mathrm{As}$ and $\mathrm{Hg}$ may adversely affect the irrigated plants, wastewater treatment system and existing biota of receiving water bodies (Pandey, 2006; Singh and Agrawal, 2010). Metal pollution adversely affects the density and diversity of biotic communities including the human race (Mountouris et al., 2002).

Table 4 : Physico-chemical characteristics of industrial effluent at Cluster-D (CD) at SIIDCUL-IIE, Haridwar during the year 2013-2014

\begin{tabular}{|c|c|c|c|c|c|c|c|c|c|c|c|c|c|}
\hline Parameter & Feb & Mar & Apr & May & June & July & Aug & Sep & Oct & Nov & Dec & Jan & BIS \\
\hline $\mathrm{pH}$ & $\begin{array}{l}7.73 \\
\pm 0.47\end{array}$ & $\begin{array}{l}7.43 \\
\pm 0.53\end{array}$ & $\begin{array}{l}7.45 \\
\pm 0.52\end{array}$ & $\begin{array}{l}7.25 \\
\pm 0.35\end{array}$ & $\begin{array}{l}5.75 \\
\pm 0.56\end{array}$ & $\begin{array}{l}5.56 \\
\pm 0.51\end{array}$ & $\begin{array}{l}7.63 \\
\pm 0.76\end{array}$ & $\begin{array}{l}7.69 \\
\pm 0.0\end{array}$ & $\begin{array}{l}6.79 \\
\pm 0.02\end{array}$ & $\begin{array}{l}7.6 \\
\pm 0.15\end{array}$ & $\begin{array}{l}7.62 \\
\pm 0.03\end{array}$ & $\begin{array}{l}7.35 \\
\pm 0.67\end{array}$ & $5.5-9$ \\
\hline Temp & $\begin{array}{l}25.57 \\
\pm 0.47\end{array}$ & $\begin{array}{l}26.3 \\
\pm 0.53\end{array}$ & $\begin{array}{l}28.1 \\
\pm 0.82\end{array}$ & $\begin{array}{l}30.3 \\
\pm 1.35\end{array}$ & $\begin{array}{l}28.97 \\
\pm 0.58\end{array}$ & $\begin{array}{l}29.97 \\
\pm 1.59\end{array}$ & $\begin{array}{l}29.73 \\
\pm 0.76\end{array}$ & $\begin{array}{l}29.3 \\
\pm 0.0\end{array}$ & $\begin{array}{l}29.07 \\
\pm 1.02\end{array}$ & $\begin{array}{l}24.4 \\
\pm 2.05\end{array}$ & $\begin{array}{l}25.77 \\
\pm 1.03\end{array}$ & $\begin{array}{l}26.37 \\
\pm 0.67\end{array}$ & \\
\hline TS & $\begin{array}{l}1200 \\
\pm 75.0\end{array}$ & $\begin{array}{l}856.67 \\
\pm 29.0\end{array}$ & $\begin{array}{l}706.67 \\
\pm 25.98\end{array}$ & $\begin{array}{l}880 \\
\pm 48.0\end{array}$ & $\begin{array}{r}1593.3 \\
\pm 91.65\end{array}$ & $\begin{array}{l}740 \\
\pm 26.0\end{array}$ & $\begin{array}{l}800 \\
\pm 52.87\end{array}$ & $\begin{array}{l}543.33 \\
\pm 19.0\end{array}$ & $\begin{array}{l}1063.3 \\
\pm 47.0\end{array}$ & $\begin{array}{l}826.67 \\
\pm 16.0\end{array}$ & $\begin{array}{l}933.33 \\
\pm 40.0\end{array}$ & $\begin{array}{l}693.33 \\
\pm 18.50\end{array}$ & \\
\hline TSS & $\begin{array}{l}819 \\
\pm 76.57\end{array}$ & $\begin{array}{l}175.33 \\
\pm 9.90\end{array}$ & $\begin{array}{l}109.33 \\
\pm 9.84\end{array}$ & $\begin{array}{l}127 \\
\pm 8.02\end{array}$ & $\begin{array}{l}96 \\
\pm 8.18\end{array}$ & $\begin{array}{r}208.33 \\
\pm 14.40\end{array}$ & $\begin{array}{l}273 \\
\pm 12.60\end{array}$ & $\begin{array}{l}108.67 \\
\pm 5.0\end{array}$ & $\begin{array}{l}341.67 \\
\pm 19.81\end{array}$ & $\begin{array}{l}362.67 \\
\pm 25.79\end{array}$ & $\begin{array}{l}268 \\
\pm 18.66\end{array}$ & $\begin{array}{l}187.33 \\
\pm 9.19\end{array}$ & . \\
\hline TDS & $\begin{array}{l}381 \\
\pm 15.1\end{array}$ & $\begin{array}{l}681.33 \\
\pm 58.3\end{array}$ & $\begin{array}{l}597.33 \\
\pm 21.70\end{array}$ & $\begin{array}{l}753 \\
\pm 54.30\end{array}$ & $\begin{array}{l}1497.3 \\
\pm 17.1\end{array}$ & $\begin{array}{l}531.67 \\
\pm 23.8\end{array}$ & $\begin{array}{l}604.33 \\
\pm 43.20\end{array}$ & $\begin{array}{l}434.67 \\
\pm 21.22\end{array}$ & $\begin{array}{l}341.67 \\
\pm 19.81\end{array}$ & $\begin{array}{l}362.67 \\
\pm 25.79\end{array}$ & $\begin{array}{l}268 \\
\pm 18.66\end{array}$ & $\begin{array}{l}187.33 \\
\pm 5.19\end{array}$ & 500 \\
\hline Turbidity & $\begin{array}{l}36 \\
\pm 1.45\end{array}$ & $\begin{array}{l}118.67 \\
\pm 4.11\end{array}$ & $\begin{array}{l}14.67 \\
\pm 0.51\end{array}$ & $\begin{array}{l}6.0 \\
\pm 0.46\end{array}$ & $\begin{array}{l}12.63 \\
\pm 0.55\end{array}$ & $\begin{array}{l}6.67 \\
\pm 0.50\end{array}$ & $\begin{array}{l}3.5 \\
\pm 0.29\end{array}$ & $\begin{array}{l}3.33 \\
\pm 1.30\end{array}$ & $\begin{array}{l}73.5 \\
\pm 3.89\end{array}$ & $\begin{array}{l}17.13 \\
\pm 0.36\end{array}$ & $\begin{array}{l}5.26 \\
\pm 0.25\end{array}$ & $\begin{array}{l}4.66 \\
\pm 0.08\end{array}$ & \\
\hline DO & $\begin{array}{l}0.1 \\
\pm 0.01\end{array}$ & $\begin{array}{l}1.93 \\
\pm 0.05\end{array}$ & $\begin{array}{l}3.8 \\
\pm 0.18\end{array}$ & $\begin{array}{l}1.83 \\
\pm 0.07\end{array}$ & $\begin{array}{l}3.5 \\
\pm 0.02\end{array}$ & $\begin{array}{l}2.77 \\
\pm 0.03\end{array}$ & $\begin{array}{l}3.93 \\
\pm 0.12\end{array}$ & $\begin{array}{l}3.03 \\
\pm 0.19\end{array}$ & Nil & $\begin{array}{l}2.26 \\
\pm 0.17\end{array}$ & $\begin{array}{l}19.63 \\
\pm 0.85\end{array}$ & $\begin{array}{l}1.2 \\
\pm 0.10\end{array}$ & \\
\hline BOD & $\begin{array}{l}117.0 \\
\pm 10.0\end{array}$ & $\begin{array}{l}326.67 \\
\pm 15.42\end{array}$ & $\begin{array}{l}190.87 \\
\pm 12.24\end{array}$ & $\begin{array}{l}173.33 \\
\pm 12.41\end{array}$ & $\begin{array}{l}245.6 \\
\pm 2.78\end{array}$ & $\begin{array}{l}203.33 \\
\pm 16.258\end{array}$ & $\begin{array}{l}266.67 \\
\pm 4.23\end{array}$ & $\begin{array}{l}243.33 \\
\pm 13.62\end{array}$ & $\begin{array}{l}173.33 \\
\pm 5.09\end{array}$ & $\begin{array}{l}109.67 \\
\pm 1.50\end{array}$ & $\begin{array}{l}193.33 \\
\pm 3.16\end{array}$ & $\begin{array}{l}206.67 \\
\pm 9.70\end{array}$ & 30 \\
\hline COD & $\begin{array}{l}573.33 \\
\pm 49.37\end{array}$ & $\begin{array}{l}430 \\
\pm 18.17\end{array}$ & $\begin{array}{l}213.33 \\
\pm 11.55\end{array}$ & $\begin{array}{l}346.67 \\
\pm 26.53\end{array}$ & $\begin{array}{l}270 \\
\pm 13.75\end{array}$ & $\begin{array}{l}290.67 \\
\pm 14.73\end{array}$ & $\begin{array}{l}340 \\
\pm 21.54\end{array}$ & $\begin{array}{l}321.67 \\
\pm 28.13\end{array}$ & $\begin{array}{l}218.33 \\
\pm 4.21\end{array}$ & $\begin{array}{l}216.67 \\
\pm 9.39\end{array}$ & $\begin{array}{l}283.33 \\
\pm 15.75\end{array}$ & $\begin{array}{l}330 \\
\pm 8.82\end{array}$ & 250 \\
\hline $\mathrm{HCO}_{3}^{-}$ & $\begin{array}{l}750.3 \\
\pm 63.23\end{array}$ & $\begin{array}{l}439.07 \\
\pm 40.13\end{array}$ & $\begin{array}{l}325.3 \\
\pm 27.197\end{array}$ & $\begin{array}{r}392.43 \\
\pm 19.68\end{array}$ & $\begin{array}{l}309.07 \\
\pm 3.04\end{array}$ & $\begin{array}{l}225.7 \\
\pm 16.48\end{array}$ & $\begin{array}{l}158.63 \\
\pm 10.30\end{array}$ & $\begin{array}{l}183.7 \\
\pm 9.95\end{array}$ & $\begin{array}{l}288.73 \\
\pm 7.46\end{array}$ & $\begin{array}{l}315.16 \\
\pm 7.77\end{array}$ & $\begin{array}{l}284.66 \\
\pm 21.13\end{array}$ & $\begin{array}{l}164.7 \\
\pm 3.69\end{array}$ & \\
\hline Hardness & $\begin{array}{l}1000 \\
\pm 62.82\end{array}$ & $\begin{array}{l}560 \\
\pm 13.30\end{array}$ & $\begin{array}{l}600 \\
\pm 40.18\end{array}$ & $\begin{array}{l}493.33 \\
\pm 23.09\end{array}$ & $\begin{array}{l}520 \\
\pm 49.28\end{array}$ & $\begin{array}{l}473.33 \\
\pm 30.23\end{array}$ & $\begin{array}{l}493.33 \\
\pm 46.18\end{array}$ & $\begin{array}{l}463.33 \\
\pm 26.86\end{array}$ & $\begin{array}{l}680 \\
\pm 10.00\end{array}$ & $\begin{array}{l}543.33 \\
\pm 20.81\end{array}$ & $\begin{array}{l}613.33 \\
\pm 23.09\end{array}$ & $\begin{array}{l}500 \\
\pm 20.00\end{array}$ & \\
\hline Acidity & $\begin{array}{l}139.17 \\
\pm 13.77\end{array}$ & $\begin{array}{l}143.33 \\
\pm 7.77\end{array}$ & $\begin{array}{l}112.5 \\
\pm 15.14\end{array}$ & $\begin{array}{l}104.17 \\
\pm 6.02\end{array}$ & $\begin{array}{l}108.33 \\
\pm 6.37\end{array}$ & $\begin{array}{l}100 \\
\pm 0.0\end{array}$ & $\begin{array}{l}55 \\
\pm 2.11\end{array}$ & $\begin{array}{l}89.83 \\
\pm 2.25\end{array}$ & $\begin{array}{l}83.333 \\
\pm 6.86\end{array}$ & $\begin{array}{l}83.333 \\
\pm 6.65\end{array}$ & $\begin{array}{l}22.5 \\
\pm 1.61\end{array}$ & $\begin{array}{l}79.167 \\
\pm 4.66\end{array}$ & \\
\hline Alkalinity & $\begin{array}{l}783.33 \\
\pm 20.83\end{array}$ & $\begin{array}{l}683.33 \\
\pm 55.38\end{array}$ & $\begin{array}{l}600 \\
\pm 40.0\end{array}$ & $\begin{array}{l}633.33 \\
\pm 28.88\end{array}$ & $\begin{array}{l}466.67 \\
\pm 27.23\end{array}$ & $\begin{array}{l}550 \\
\pm 26.603\end{array}$ & $\begin{array}{c}566.67 \\
3 \pm 36.37\end{array}$ & $\begin{array}{l}89.83 \\
\pm 8.59\end{array}$ & $\begin{array}{l}500 \\
\pm 836.603\end{array}$ & $\begin{array}{l}413.33 \\
3\end{array}$ & $\begin{array}{l}620 \\
\pm 23.09\end{array}$ & $\begin{array}{l}600 \\
\pm 20.0\end{array}$ & $\overline{ \pm 50.0}$ \\
\hline K & $\begin{array}{l}5.13 \\
\pm 0.16\end{array}$ & $\begin{array}{l}15.2 \\
\pm 0.1\end{array}$ & $\begin{array}{l}18.9 \\
\pm 4.45\end{array}$ & $\begin{array}{l}25 \\
\pm 12.50\end{array}$ & $\begin{array}{l}11.3 \\
\pm 5.39\end{array}$ & $\begin{array}{l}9.83 \\
\pm 4.14\end{array}$ & $\begin{array}{l}11.1 \\
\pm 2.64\end{array}$ & $\begin{array}{l}3.87 \\
\pm 0.28\end{array}$ & $\begin{array}{l}7.43 \\
\pm 0.41\end{array}$ & $\begin{array}{l}4.33 \\
\pm 0.38\end{array}$ & $\begin{array}{l}21.2 \\
\pm 1.80\end{array}$ & $\begin{array}{l}4.1 \\
\pm 1.49\end{array}$ & . \\
\hline$P$ & $\begin{array}{l}3.0 \\
\pm 0.0\end{array}$ & $\begin{array}{l}0.83 \\
\pm 0.09\end{array}$ & $\begin{array}{l}4.0 \\
\pm .01\end{array}$ & $\begin{array}{l}15.5 \\
\pm 0.47\end{array}$ & $\begin{array}{l}5.17 \\
\pm 0.05\end{array}$ & $\begin{array}{l}0.83 \\
\pm 0.04\end{array}$ & $\begin{array}{l}13.3 \\
\pm 1.11\end{array}$ & $\begin{array}{l}0.18 \\
\pm 0.09\end{array}$ & $\begin{array}{l}7.17 \\
\pm 0.51\end{array}$ & $\begin{array}{l}4.53 \\
\pm 0.29\end{array}$ & $\begin{array}{l}6.77 \\
\pm 0.46\end{array}$ & $\begin{array}{l}7.9 \\
\pm 0.59\end{array}$ & 5 \\
\hline
\end{tabular}

All parameters are expressed in $\mathrm{mg} \mathrm{I}^{-1}$ except $\mathrm{pH}$, temperature $\left({ }^{0} \mathrm{C}\right.$ ) and turbidity (NTU); BIS: Bureau of Indian Standards for inland disposal of effluents; Vlaues are mean \pm SD 
Unlike some organic pesticides, metals cannot be broken down into less harmful components in the environment (Lenntech, 2007) and thus, are responsible for building up concentration upto the harmful levels which may damage environment.

In Indian industrial sector, traditional production methodologies, weaknesses in recycling and/or improper disposal of industrial waste and weak implementation of environmental policies have lead to increased heavy metal concentration up to alarming level (Kaur et al., 2012). In the present study, heavy metals viz., Cd (27.53 $\left.\mathrm{mg} \mathrm{l}^{-1}\right), \mathrm{Cr}\left(11.25 \mathrm{mg} \mathrm{l}^{-1}\right), \mathrm{Cu}\left(4.9 \mathrm{mg} \mathrm{l}^{-1}\right), \mathrm{Fe}$ (37.38 $\left.\mathrm{mg} \mathrm{l}^{-1}\right), \mathrm{Mn}\left(2.12 \mathrm{mgl}^{-1}\right), \mathrm{Ni}\left(15.3 \mathrm{mg} \mathrm{l}^{-1}\right), \mathrm{Pb}\left(2.11 \mathrm{mgl}^{-1}\right)$ and $\mathrm{Zn}\left(21.26 \mathrm{mg} \mathrm{I}^{-1}\right)$ in the composite effluents of different sites of SIIDCUL-IE were beyond the standards prescribed by BIS (2012) (Figs. 2a-h). The maximum concentration of $\mathrm{Cu}\left(4.9 \mathrm{mgl}^{-1}\right)$ (Fig. 2a) and $\mathrm{Pb}\left(2.11 \mathrm{mgl}^{-1}\right)$ (Fig. 2h) in effluents were recorded at Cluster $\mathrm{C}$ and $B$ in the month of January and May, while the concentration of $\mathrm{Cu}$ and $\mathrm{Pb}$ was below detection limit at Cluster $\mathrm{D}$ in October (Fig. 2a) and January (Fig. 2h), respectively. The concentration of Mn $\left(2.12 \mathrm{mg} \mathrm{l}^{-1}\right)$ (Fig. 2d) was found maximum at Cluster B in the month of September, while minimum concentration of $\mathrm{Mn}\left(0.01 \mathrm{mg} \mathrm{I}^{-1}\right)$ (Fig. 2d) was observed at Cluster B in October. The maximum value of $\mathrm{Fe}\left(37.38 \mathrm{mg} \mathrm{l}^{-1}\right)$ (Fig. 2c) in effluents was recorded at Cluster $\mathrm{C}$ in December, at the same time minimum value of $\mathrm{Fe}$ (1.7 $\mathrm{mg}^{-1}$ ) (Fig. 2c) was recorded at Cluster A in the month of October. Singh et al. (2012) assessed heavy metals profile of wastewater discharged from an industrial area at Dehradun, India and reported approximately similar values for $\mathrm{Cu}\left(3.78 \mathrm{mg} \mathrm{l}^{-1}\right)$ but higher values for $\mathrm{Pb}\left(5.23 \mathrm{mg} \mathrm{l}^{-1}\right)$ and $\mathrm{Mn}\left(7.45 \mathrm{mgl}^{-1}\right)$. The concentration of Fe in SIIDCUL-IE was found to be notably higher than the values (19.38mg/l) reported by Sankpal and Naikwade (2012) in pharmaceutical industries effluent at Lote Pershuram industrial area, Ratnagiri, India.

At SIIDCUL-IE, maximum concentration of $\mathrm{Cr}\left(11.25 \mathrm{mg} \mathrm{I}^{-1}\right)$ (Fig. 2g) in composite effluents was observed at Cluster $C$ in the month of December i.e., beyond the permissible limit of BIS, while minimum ( $\left.0.23 \mathrm{mg} \mathrm{l}^{-1}\right)$ (Fig. 2g) was observed at the same Cluster in the month of January. In contrast, Azeem (2009) reported relatively higher $\left(22.27 \mathrm{mg} \mathrm{l}^{-1}\right)$ concentration for $\mathrm{Cr}$ in industrial wastewater at Lahore, Pakistan. Similarly, Singare and Dhabarde (2014) reported exceedingly higher (than prescribed limits) values for $\mathrm{Cr}$ from Fine chemical manufacturing industries $\left(4.73 \mathrm{mg} \mathrm{l}^{-1}\right)$ and metallurgical units $\left(2.11 \mathrm{mg} \mathrm{I}^{-1}\right)$ at Mumbai, India. Similar observations of exceedingly higher (than permissible standards) concentration of elements like $\mathrm{Cu}\left(7.97 \mathrm{mg} \mathrm{l}^{-1}\right), \mathrm{Fe}\left(8.32 \mathrm{mg} \mathrm{l}^{-1}\right), \mathrm{Mn}$ $\left(1.24 \mathrm{mg} \mathrm{l}^{-1}\right)$ and $\mathrm{Pb}\left(1.10 \mathrm{mg} \mathrm{I}^{-1}\right)$ were reported by Adakole and Abolude (2009) in the effluents discharged from the industrial units involved in metal finishing works at Nigeria It is important to note that Cluster $\mathrm{C}$ at SIIDCUL-IE had maximum number of industrial installations engaged in metal-related works, pharmaceuticals, chemicals, food packaging material, textile made-ups, aluminum and zinc based alloys. This might be a probable reason that the metals utilized in alloys particularly $\mathrm{Cu}, \mathrm{Pb}, \mathrm{Mn}, \mathrm{Fe}$ and $\mathrm{Cr}$ are discharged in the effluents and are beyond the permissible limits at a time when these industries are in their operation mode at Cluster $\mathrm{C}$ of the area. The maximum concentration of $\mathrm{Zn}\left(21.26 \mathrm{mgl}^{-1}\right)$ (Fig. 5) in SIIDCUL-IE industrial effluents was recorded at Cluster B in the month of November, whereas minimum concentration of $\mathrm{Zn}$ (0.681 $\mathrm{mg} \mathrm{I}^{-1}$ ) (Fig. 5) was observed at Cluster $\mathrm{C}$ in the same month. The observed values were much higher than the concentration range of $\mathrm{Zn}\left(0.35-1.34 \mathrm{mg} \mathrm{I}^{-1}\right)$ reported by Ramola and Singh (2013) in a study of assessment of heavy metal profile of pharmaceutical industry effluents of Dehradun, India. Kumar and Thatheyus (2013) reported exceedingly higher values for Zn (739 $\mathrm{mg} \mathrm{I}^{-1}$ ) than the permissible standards in a study of effluents discharged from electroplating units at Madurai, India. It is important to note that Cluster B had factories dealing with the production of electronics and electrical items, brass and zinc ingots, floor ceramic tiles and glass items, automobile parts, fabrication of steel, multilayer film, fire extinguishing formulations, aluminum anodizing, $\mathrm{Zn}$ and Ni plating, which might be a possible reason for considerably higher concentration of $\mathrm{Zn}$ in industrial effluents at SIIDCUL Industrial complex.

The maximum values of $\mathrm{Cd}\left(27.53 \mathrm{mg} \mathrm{l}^{-1}\right)$ (Fig. 2e) and $\mathrm{Ni}$ $\left(15.3 \mathrm{mg} \mathrm{l}^{-1}\right)$ (Fig. 2f) were observed at Cluster $D$ in the month of October, whereas minimum values of $\mathrm{Cd}\left(0.0163 \mathrm{mg} \mathrm{l}^{-1}\right)$ (Fig. 2e) and $\mathrm{Ni}\left(0.087 \mathrm{mg} \mathrm{l}^{-1}\right)$ (Fig. 2f) were recorded at Cluster $\mathrm{A}$ in November and Cluster $\mathrm{C}$ in the month of January, respectively. The concentration of $\mathrm{Cd}\left(0.74 \mathrm{mgl}^{-1}\right)$ observed in the effluents was considerably higher than the values of $\mathrm{Cd}$ reported by Joshi and Santani (2012) in a study for quality assessment of effluent discharged from textile industry at Vapi, India. The observed value of $\mathrm{Ni}$ in the effluents was also much higher than the values of $\mathrm{Ni}\left(0.935 \mathrm{mg} \mathrm{l}^{-1}\right)$ reported by James et al. (2013) in pharmaceutical industry effluents of Ogun State, Nigeria. At Cluster D, cadmium and nickel were beyond the permissible limits of BIS (2012). The industries running in this Cluster deal with engineering operations like manufacturing of welding cables, stainless steel furniture, pre-fabricated steel, cotton and synthetic yarn, transformers products, dry cells, ferrous and non ferrous metal, fast moving consumer goods (FMCG) such as detergents, soaps, hair care etc. In reference to the quantum of heavy metals released from type of industry running in the particular area, Sanyaolu et al. (2013) reported exceedingly higher (beyond the prescribed limits of WHO) concentration of nickel $\left(0.093 \mathrm{mg} \mathrm{l}^{-1}\right)$ in the effluent of FMCG products manufacturing industry at lkorodu, Lagos State, Nigeria, while Lokhande et al. (2011) reported exceedingly higher (beyond the permissible limits of BIS concentration of cadmium (26.0 $\mathrm{mg} \mathrm{l}^{-1}$ ) in the effluents discharged from metal related engineering work industries at Mumbai, India. The values of $\mathrm{Cu}$, $\mathrm{Cr}$ and $\mathrm{Pb}$ reported in present study were notably higher than those observed by Donatus (2016) [Cu:1.09 $\mathrm{mg} \mathrm{l}^{-1}$, Cr: 2.20, Pb: 2.97 and $\mathrm{Cd}$ : 0.25] in the industrial effluents at Vellore, Tamil Nadu, while Islam et al. (2016) also reported quite lower values of 


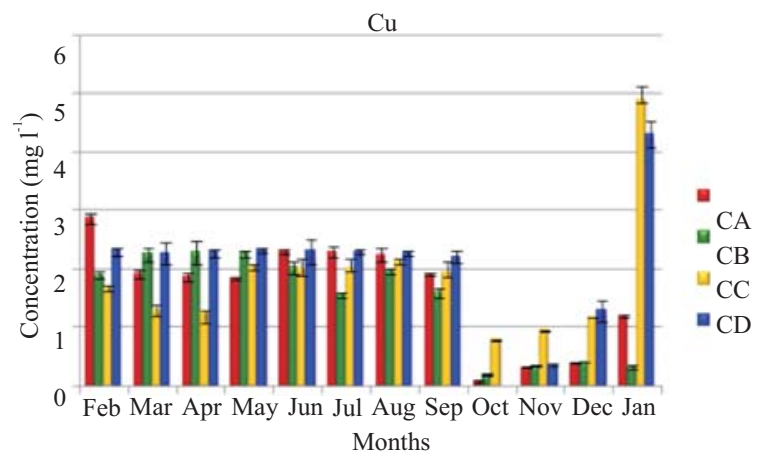

Fig. 2a : Monthly variation of $\mathrm{Cu}$ at four clusters in SIDCUL-IE, Haridwar during the year 2013-2014 (Mean \pm SD of three values)

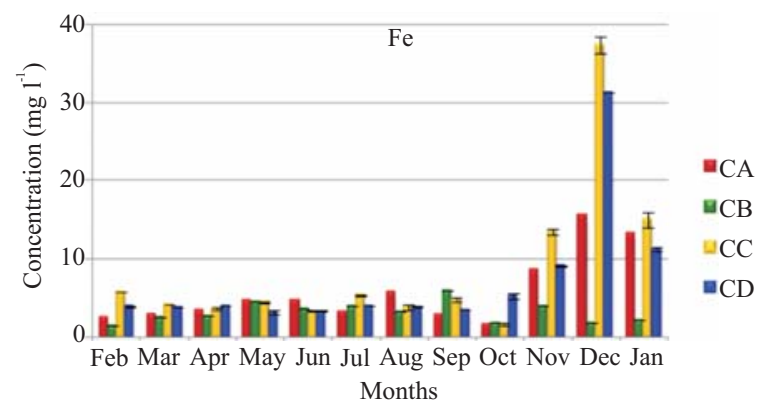

Fig. 2c : Monthly variation of Fe at four clusters in SIDCUL-IE, Haridwar during the year 2013-2014 (Mean \pm SD of three values)

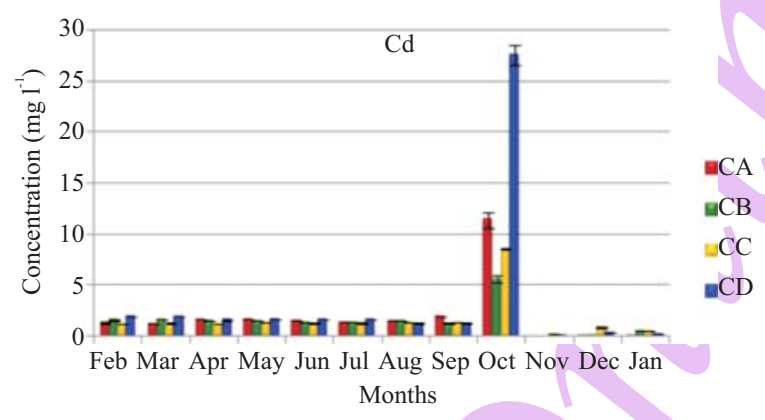

Fig. 2e : Monthly variation of $\mathrm{Cd}$ at four clusters in SIDCUL-IE, Haridwar during the year 2013-2014 (Mean \pm SD of three values)

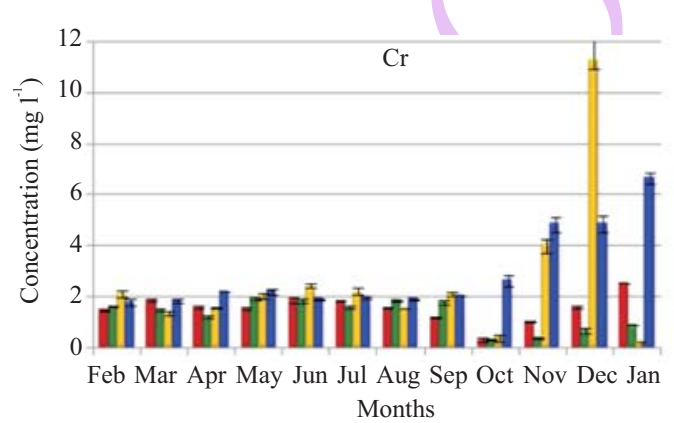

Fig. 2g : Monthly variation of $\mathrm{Cr}$ at four clusters in SIDCUL-IE, Haridwar during the year 2013-2014 (Mean \pm SD of three values)

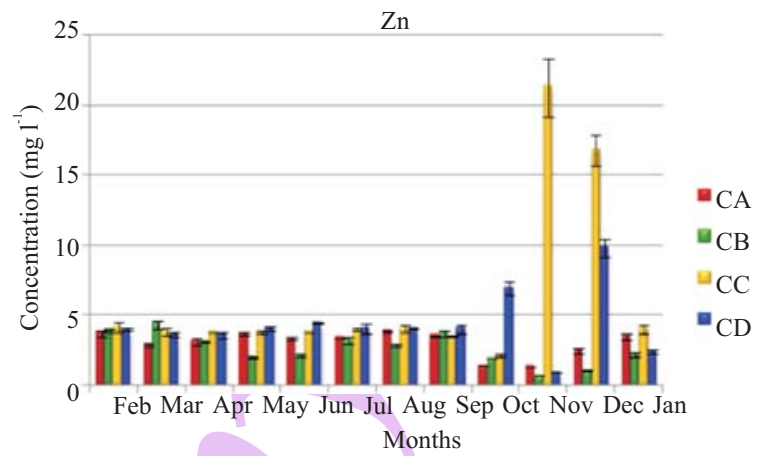

Fig. 2b : Monthly variation of $\mathrm{Zn}$ at four clusters in SIDCUL-IE, Haridwar during the year 2013-2014 (Mean \pm SD of three values)

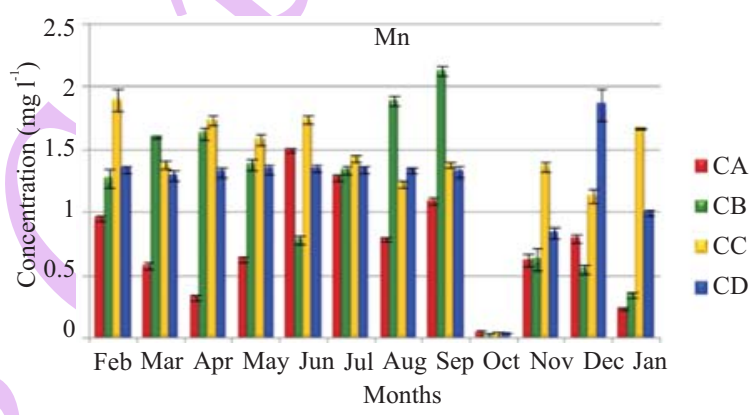

Fig. 2d : Monthly variation of Mn at four clusters in SIDCUL-IE, Haridwar during the year 2013-2014 (Mean \pm SD of three values)

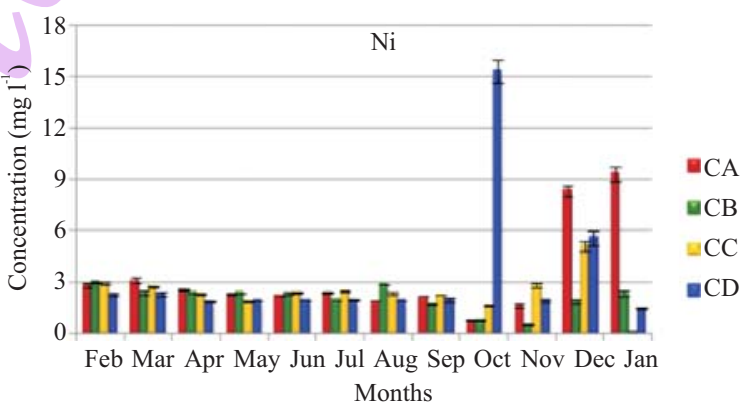

Fig. 2f : Monthly variation of Ni at four clusters in SIDCUL-IE, Haridwar during the year 2013-2014 (Mean \pm SD of three values)

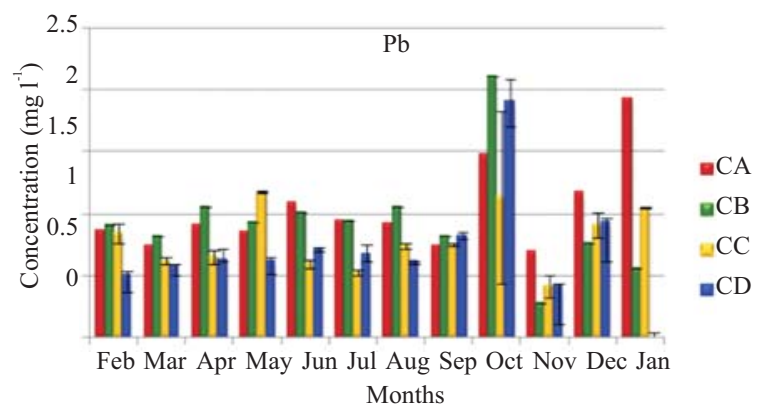

Fig. 2h : Monthly variation of $\mathrm{Pb}$ at four clusters in SIDCUL-IE, Haridwar during the year 2013-2014 (Mean \pm SD of three values) 
$\mathrm{Cu}(1.58)$ and $\mathrm{Mn}$ (0.68) in the effluents of Kushtia Industrial zone in Bangladesh. Therefore, higher content of $\mathrm{Ni}$ and $\mathrm{Cd}$ in the effluents might probably be due to FMCG and the engineering units. However, during this study, a trend of notably higher concentration of trace metals was observed during the month of November and December. This might be due to an increase in production process that was proportionate to the increase in demand of the manufactured goods in consumer markets.

The present study observed monthly variations of all the physicochemical and metallic variables in the composite Industrial effluents of different Clusters (A, B, C and D) at SIIDCUL-IE. Variations in the variables might be due to the operational mode of the industries for production of the products depending on the consumer demand. Karl Pearson's correlation matrix was calculated for physico-chemical and heavy metals variables to find out the relationship (if any) among various variables. Different parameters showed significant (either positive or negative) relationship with each other. The variables showed strong to moderate, either positive or negative correlation with each other. Among various significantly correlated variables, $\mathrm{pH}$-acidity at Cluster A and B; and potassium and acidity at Cluster B and C were found to significantly correlated with each other, while metals like $\mathrm{Cr}$, Ni, Fe, Cu and $\mathrm{Zn}$ were found to be significantly positive correlated at either of the Clusters. It is important to note that $\mathrm{Cr}$ and $\mathrm{Ni}$ showed a strong positive correlation with each other at Cluster $A(r=0.622), B(r=0.807)$ and $C(r=0.860)$. These three clusters have numerous factories dealing with the works related to pharmaceuticals, iron, steel and electroplating of $\mathrm{Zn}$ and Ni which might be a probable reason for higher concentration of $\mathrm{Cr}$ and $\mathrm{Ni}$.

It was concluded that the industrial effluents discharged from SIIDCUL-IE had relatively poor quality as per standards of $\mathrm{BIS}$. There were monthly fluctuations in various physic-chemical parameters and metallic concentrations of the effluents which may be due to the discharge of pollutants in varying concentrations. The trend of concentration of metals in the effluent of different sampling sites in the order $\mathrm{Fe}>\mathrm{Cd}>\mathrm{Zn}>\mathrm{Ni}>\mathrm{Cr}>\mathrm{Cu}>\mathrm{Pb}>\mathrm{Mn}$ may possibly be due to more number of units dealing with iron and steel facility.

\section{Acknowledgment}

The authors acknowledge the Department of Science and Technology, Government of India for sanctioning the research project (File no: DST/TM/WTI/2K12/34) and financial assistance in the course of study under this project.

\section{References}

Abraha, K., A. Gebrekidan, Y. Weldegebrie and A. Hadera: Physicochemical analysis of Almeda Textile Industry effluents in Tigray, Northern Ethiopia. J. Environ. Anal. Chem., 1, 1-7 (2014).
Adakole, J.A. and D.S. Abolude: Studies on effluent characteristics of a metal finishing company, Zaria-Nigeria. Res. J. Environ. Earth Sci., 1,54-57 (2009).

Ahmad, M.T., M. Sushil and M. Krishna: Influence of dye industrial effluent on physico chemical characteristics properties of soil at Bhairavgarh, Ujjain, MP, India. Int. Res. J. Env. Sci., 1, 50-53 (2012).

Al-Farraj, A.S., M. Al-Sewailem, A. Aly, M. Al-Wabel and S. El- Maghraby: Assessment and heavy metal behaviors of industrial waste water: A case study of Riyadh city, Saudi Arabia. Procee. Inter. Acad. Ecol. Environ. Sci., 3, 266-277 (2013).

APHA: Standard methods of Water and Wastewater Analysis $22^{\text {th }}$ Edn., American Public Health Association, Washington DC, USA(2012).

Arora, T., A. Mishra, G. Matta, A.K. Chopra, A. Kumar, D.R. Khanna and V. Kumar: Human health risk assessment of temporal and spatial variations of ground water quality at a densely industrialized commercial complex at Haridwar, India. J. Appl. Nat. Sci., 6, 825843 (2014).

Azeem, H.A.: Analysis of industrial waste water from Kot Lakhpat area (Lahore, Pakistan) by atomic absorption spectrometer. Biologia (Pakistan), 55, 35-41 (2009).

Bae, M.J., J.H. Chun, T.S. Chon and Y.S. Park: Spatio-temporal variability in benthic macroinvertebrate communities in headwater streams in South Korea. Water, 8, 1-15(2016).

Bharti, P.K., P. Kumar and V. Singh: Impact of industrial effluents on ground water and soil quality in the vicinity of industrial area of Panipat city, India. J. Appl. Nat. Sci., 5, 132-136 (2013).

Bureau of Indian Standard (BIS): General standards for discharge of environmental pollutants part-A: effluents. (IS: 10500) http://hppcb.gov.in/eiasorang/spec.pdf. (1991)

CGWB: Central Groundwater Board of India, Technical Report, 2002, p. $38(2002)$.

Cuevas, J., A.I. Ruiz, I.S. Soto, T. Sevilla, J.R. Procopio, P. Da Silva, M.J. Gismera, M. Regadío, J.N. Sánchez, R.M. Rodríguez and S. Leguey: The performance of natural clay as a barrier to the diffusion of municipal solid waste landfill leachates. J. Env. Manag., 95, 175-181 (2011).

Chuma, F.M., M.M. Kelvin and N.N. Karoli: Assessment of heavy metals in treated wastewater used for the irrigation of vegetable plants in Arusha City. Int. J. Res. Chem. Environ., 5, 54-60 (2014).

Dutta, K. and A.R. Ghosh: Analysis of physico-chemical characteristics and metals in water sources of chromite mining in Sukinda Valley, Odisha, India. J. Environ. Biol., 34, 783-788 (2013).

Donatus, M.: Removal of heavy metals from industrial effluent using Salvinia molesta. Int. J. Chem. Tech. Res., 9, 608-613 (2016).

Goel, P.K.: Water pollution, causes, effects and control. New Age International (P) Ltd., Publishers, New Delhi (1997).

Haydar, S., G. Hussain, H. Haider and A. Rehman: Wastewater characterization of selected industries in Quaid-e-Azam Industrial Estate: Treatment options and impact on groundwater quality. In: Proceedings of the Pakistan Academy of Sciences, 2, 93-102 (2016).

Idris, M.A., B.G. Kolo, S.T. Garba and I. Waziri: Pharmaceutical industrialeffluent: Heavy metal contamination of surface water in Minna, Niger State, Nigeria. Bull. Env. Pharmacol. Life Sci., 2, 4044 (2013).

Islam, M.J., M.A. Hakim, M.M. Hanafi, A.S. Juraimi, S. Aktar, A. Siddiqa, Rahman, M.A. Islam and M.A. Halim: Hydrogeochemical quality 
and suitability studies of groundwater in northern Bangladesh. $\mathrm{J}$. Environ. Biol., 35, 765-779 (2014).

Islam, R., J. Al. Foisal, M. Rahman, L. A. Lisa and D. K. Paul: Pollution assessment and heavy metal determination by AAS in waste water collected from Kushtia industrial zone in Bangladesh. Afric. J. Environ. Sci. Technol., 10, 9-17 (2016).

Islam, M.M., K. Mahmud, O. Faruk and M.S. Billah: Textile dyeing industries in Bangladesh for sustainable development. IJESD, 2, 428-436 (2011)

Jadhav, B.V. and H.N. Nandan: Impact of industrial effluents in the physico-chemical parameters of ground water of Dhule MIDC (India) Area. Int. J. Rec. Innov. Trends Comput. Comm., 4, 659662 (2016).

Jamaluddin, A.M. and M. Nizamuddin: Physico-chemical assessment of textile effluents in chittagong region of Bangladesh and their possible effects on environment. Int. J. Res. Chem. Environ., 2, 220-230 (2012).

James, O.O., K. Nwaeze, E. Mesagan, M. Agbojo, K.L. Saka and D.J. Olabanji: Concentration of heavy metals in five pharmaceutical effluents in Ogun State, Nigeria. Bull. Env. Pharmacol. Life Sci., 2, 84-90 (2013).

Joshi, V.J. and D.D. Santani: Physico-chemical characterization and heavy metal concentration in effluent of textile industry. Univ. $J$. Env. Res. Tech., 2, 93-96 (2012).

Katiyar, S. and D. Singh: Prevalence of arsenic exposure in population of Ballia district from drinking water and its correlation with blood arsenic level. J. Environ. Biol., 35, 589-594 (2014).

Kaur, R., S. Wani, A. Singh and K. Lal: Wastewater production, treatment and use in India. Retertived on October 21, 2015: http://www.ais.unwater.org/ais/pluginfile.php/356/mod_page/cont ent/111/CountryReport_India.pdf(2012).

Kumar, G.S. and A.J. Thatheyus: Bioremediation of chromium nickel and zinc in electroplating effluent by Escherichia coli. Ann. Rev. Res. Biol., 3, 913-920 (2013).

Kumar, R., R.D. Singh and K.D. Sharma: Water Resources India. Curr. Sci., 89, 794-811 (2005).

Krishna, C.V.: Rapid economic growth and industrialization in India, China and Brazil: at what cost? William Davidson Institute Working PaperNumber 897 (2007).

Lenntech: Water treatment and air purification. Rotterdamseweg, Netherlands (2004).

Lenntech: Water treatment and air purification. Rotterdamseweg, Netherlands (2007).

Lokhande, R.S., P.U. Singare and D.S. Pimple: Study on physicochemical parameters of waste water effluents from Taloja Industrial Area of Mumbai, India. Int. J. Ecosystem, 1, 1-9 (2011).

Mahmoud, E. K. and A. M. Ghoneim: Effect of polluted water on soil and plant contamination by heavy metals in El-Mahla El-Kobra, Egypt., Solid Earth, 7, 703-711 (2016).

Mathie, R.T., L. Hansen, M.F. Elliott and J. Hoare: Outcomes from homeopathic prescribing in veterinary practice: a prospective, research-targeted, pilot study. Homeopathy, 96, 27-34 (2007).

Morais, S., F.G. Costa and M.L. Pereira: Heavy metals and human health. In: Environmental health- emerging issues and practice (Eds.: J. Oosthuizen), InTech., 227-246 (2012).

Mountouris, A., E. Voutsas and D. Tassios: Bioconcentration of heavy metals in aquatic environments: the importance of bioavailability. Marine Poll. Bull., 44, 1136-1141 (2002).

Neustadt, J. and S. Pieczenik: Heavy metal toxicity with emphasis on mercury. Integr. Medi., 6, 26-32 (2007).

Nickson, R., C. Sengupta, P. Mitra, S.N. Dave, A.K. Banerjee, A. Bhattacharya, S. Basu, N. Kakoti, N.S. Moorthy, M. Wasuja, M. Kumar, D.S. Mishra, A. Ghosh, D.P. Vaish, A.K. Srivastava, R.M. Tripathi, S.N. Singh, R. Prasad, S. Bhattacharya and P. Deverill: Current knowledge on the distribution of arsenic in groundwater in five states of India. J. Environ. Sci. Hlth. A. Tox. Hazard. Subst. Environ. Eng., 42, 1707-1718 (2007).

Okereke, J. N., O. I. Ogidi and K. O. Obasi: Environmental and health impact of industrial wastewater effluents in Nigeria-A Review. Int. J. Adv. Res. Biol. Sci, 3, 55-67 (2016).

Pandey, S.N.: Accumulation of heavy metals ( $\mathrm{Cd}, \mathrm{Cr}, \mathrm{Cu}, \mathrm{Ni}$ and $\mathrm{Zn}$ ) in Raphanus sativus L. and Spinacia oleracea L. plants irrigated with industrial effluent. J. Environ. Biol., 37, 381-384 (2006).

Paul, S.A., S.K. Chavan and S.D. Khambea: Studies on characterization of textile industrial waste water in Solapur City. Int. J. Chem. Sci., 10, 635-664 (2012).

Peterson, F.L. and J. Oberdorfer: Uses and abuses of wastewater injection wellsin Hawaii. Pacific Sci., 39, 230-240 (1985).

Ramola, B. and A. Singh: Heavy metal concentrations in pharmaceutical effluents of industrial area of Dehradun (Uttarakhand). Indian. J. Environ. Anal. Toxicol., 3, 173 (2013).

Raikwar, M.K., P. Kumar, M. Singh and A. Singh: Toxic effect of heavy metals in livestock health. Veterinary World, 1, 28-30 (2008).

Sahu, R.K., S. Katiyar, J. Tiwari and G.C. Kisku: Assessment of drain water receiving effluent from tanneries and its impact on soil and plants with particular emphasis on bioaccumulation of heavy metals. J. Environ. Biol., 28, 685-690 (2007).

Sankpal, S.T. and P.V. Naikwade: Heavy metal concentration in effluent discharge of pharmaceutical industries. Sci. Res. Rep., 2, 88-90 (2012).

Santona, L., P. Castaldi and P. Melis: Evaluation of the interaction mechanisms between red muds and heavy metals. J. Haz. Mater., 136, 324-329 (2006).

Sanyaolu, V.T., A.A.A. Sanyaolu and A. Babayeju: Determination of the physico-chemical parameters of an industrial effluent:Acase study of Pz Cussons Plc, Ikorodu, Lagos State. JECR, 1, $12-20$ (2013).

Singare, P.U. and S.S. Dhabarde: Studies on pollution due to discharge of effluent from pharmaceutical industries of Dombivali Industrial Belt of Mumbai, India. Euro. J. Environ. Saf. Sci., 2, 5-11 (2014).

Singh, N., D. Kumar and A.P. Sahu: Arsenic in the environment: effects on human health and possible prevention. J. Environ. Biol., 28, 359-365 (2007).

Singh, S.M., I. Varshneya and M. Nagarkoti: Assessment of physicochemical parameters of effluents of three factories of Bareilly district and their possible effects on grazing animals and cereals. J. Environ. Biol., 19, 271-274 (1998).

Singh, S.N., G. Srivastav and A. Bhatt: Physico-chemical determination of pollutants in wastewater in Dehradun. Curr. World. Environ., 7, 133-138 (2012).

Siyanbola, T.O., K.O. Ajanaku, O.O. James, J.A.O. Olugbuyiro and J.O. Adekoya: Physico-chemical characteristics of industrial effluents in Lagos State, Nigeria. Global J. Pure Appl. Sci. Technol., 1, 49-54 (2011).

Smrithi, A., T. Bhaigyabati and K. Usha: Bioremediation potential of Brassica juncea against textile disposal. Res. J. Pharm. Biol. Chem. Sci., 3, 393-400 (2012).

Surti, H. S.: Physico-chemical and microbial analysis of waste water from different industry and cod reduction treatment of industrial 
wastewater by using selective microorganisms. Int. J. Curr. Microbiol. App. Sci., 5, 707-717 (2016).

United Nations Environment Program (UNEP). Technical Report on Clearing the Waters: A focus on water quality solutions, United
Nations Environment Program (2010).

Yadav, J. and R.K. Pathak: Analysis and physico-chemical parameters of Sarvar Devla Sugar mill studies of effluents. Curr. World. Environ., 7,313-315(2012).

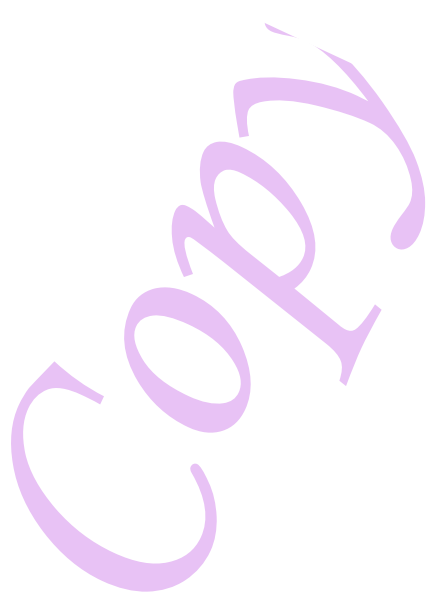

